\title{
Reference Frame Fields based on Quantum Theory Representations of Real and Complex Numbers
}

\author{
Paul Benioff, \\ Physics Division, Argonne National Laboratory, \\ Argonne, IL 60439, USA \\ e-mail:pbenioff@anl.gov
}

November 20, 2018

\begin{abstract}
A quantum theory representations of real (R) and complex (C) numbers is given that is based on states of single, finite strings of qukits for any base $k \geq 2$. Arithmetic and transformation properties of these states are given, both for basis states representing rational numbers and linear superpositions of these states. Both unary representations and the possibility that qukits with $k$ a prime number are elementary and the rest composite are discussed. Cauchy sequences of $q_{k}$ string states are defined from the arithmetic properties. The representations of $R$ and $C$, as equivalence classes of these sequences, differ from classical representations as kit string states in two ways: the freedom of choice of basis states, and the fact that each quantum theory representation is part of a mathematical structure that is itself based on the real and complex numbers. In particular, states of qukit strings are elements of Hilbert spaces, which are vector spaces over the complex field. These aspects enable the description of 3 dimensional frame fields labeled by different $\mathrm{k}$ values, different basis or gauge choices, and different iteration stages. The reference frames in the field are based on each $\mathrm{R}$ and $\mathrm{C}$ representation where each frame contains representations of all physical theories as mathematical structures based on the $\mathrm{R}$ and $\mathrm{C}$ representation. Some approaches to integrating this work with physics are described. It is observed that $\mathrm{R}$ and $\mathrm{C}$ values of physical quantities, matrix elements, etc. which are viewed in a frame as elementary and featureless, are seen in a parent frame as equivalence classes of Cauchy sequences of states of qukit strings.
\end{abstract}

\section{Introduction}

Numbers play a basic role in physics and mathematics, so basic in fact that their use, both in experiments and in theory, is taken for granted and is rarely examined. Natural numbers and integers are probably the most basic because of their role in counting, rational numbers play a basic role in that numerical experimental 
outputs are represented as rational numbers. They also are the type of numbers used in all computer computations.

The importance of real and complex numbers lies in their being the number base of all physical theories used so far. This includes classical and quantum mechanics, quantum field theory, QED, QCD, string theory, and special and general relativity. Each of these theories is a mathematical theory characterized by a different set of axioms. Assuming the axiom sets are consistent, each theory has many different representations as mathematical structures based on the real and complex numbers. The connection to physics is made by interpreting some of the elements in the mathematical structures as representing physical systems and physical quantities. Examples include the use, in quantum theory, of elements of Hilbert spaces and operators on the spaces to represent states and observable physical quantities of systems, the use of other elements, to represent various properties of space time, etc.

In all of this, the tacit assumption is made that the properties of physical systems and the physical universe are independent of the properties of mathematical theories and their representations. The general approach taken is to discover the theory that best describes physical systems and their properties. Little attention is paid to whether the basic properties of theories and their mathematical representations have any influence on the basic properties of physical systems or how intertwined physics and mathematics are.

The approach taken in this paper stems from the work of Wigner on the unreasonable effectiveness of mathematics in the natural sciences [1, 2, 3. One answer to this problem is that one should work towards developing a coherent theory of mathematics and physics together [4,5]. Presumably such a theory would show why mathematics is important to physics.

This paper is, hopefully, a step in this direction. Here extension of previous work on the quantum representation of numbers [6, 7] shows that quantum theory representations of real and complex numbers have properties not possessed by classical representations of these numbers. It will be seen that the structures resulting from these properties suggest a close intertwining between the properties of physical and mathematical systems.

Although little investigated, these possibilities are not new. Perhaps the closest is the work of Tegmark [8, 9] which suggests that the physical universe really is a mathematical structure. Other work which emphasizes the close relationship between physics and mathematics is concerned with quantum theory representations of mathematical systems. This work includes papers on quantum set theory [10, 11, 12, quantum theory representations of real numbers [13, 14, 15, 16, 17, 18, 19, and the use of category theory in physics [20, 21.

The quantum representations of real and complex numbers presented here differ from other work in this area in that they are not abstract representations based on quantum logic or on lattice valued models of set theory [13, 14, 15, 17, 18, nonstandard numbers [16], or category theory [20, 21]. Instead they are based on representations of natural numbers, $N$, integers, $I$, and rational numbers, $R a$, as states of finite strings of qukits 1

\footnotetext{
${ }^{1}$ Qukits are extensions of qubits to systems with states in a finite $k$ dimensional Hilbert space.
} 
This choice is based on the observation that all physical representations of numbers are in the form of k-ary representations as states of strings of kits or of qukits. This is the case for all experimental outputs. Also all computations are based on these representations of numbers. The importance of this type of number for computations and the limits of computation suggest other ties to information theory and limitations on the information resources of the universe [22, 23, 24. The restriction here to qukit strings is based on the fact that quantum theory is the basic underlying theory of all physical systems.

Here the quantum theory representations of real numbers are described as equivalence classes of Cauchy sequences of states of qukit strings. In essence this is a translation of the definition in mathematical analysis textbooks [25, 26] as equivalence classes of Cauchy sequences of rational numbers into quantum theory 2

These representations are described in the next two sections. First quantum representations of natural numbers, integers, and rational numbers are presented as states of single finite qukit strings. These are based on the states of each qukit as elements of a $k$ dimensional Hilbert space. These are used in the quantum representations of real numbers as equivalence classes of Cauchy sequences of states of single finite qukit strings.

Quantum representations of real and complex numbers differ from classical representations in several ways. One difference is that the equivalence classes of Cauchy sequences of qukit string states are larger than classical classes as they contain sequences that do not correspond to any classical sequence. However, no new equivalence classes are created.

A more important difference is that, for states of qukit strings, there is a freedom of basis state choice that does not exist in classical representations. This is based on the observation that the states of each qukit are elements of a $k$ dimensional Hilbert space. In order that states of qukits, $\left(q_{k}\right)$, represent numbers, one must choose a basis set of states for each $q_{k}$ in the string. This is well known in quantum computation where binary representations of numbers, such as |1100101〉 as a state of a qubit string, imply a choice of basis for each qubit. This freedom of basis choice is also referred to here as a gauge freedom or freedom to fix a gauge for each $q_{k}$. It is represented here by a variable $g$ that ranges over all basis or gauge choices for $q_{k}$ states in a string. This gauge freedom is seen to extend up to representations of real numbers in that for each gauge choice $g$ one has quantum theory representations $R_{k, g}$ of real numbers that are different for different $k$ and $g$. Even though these representations are $k, g$ dependent, they are all isomorphic to one another.

These representations for different $k$ and $g$ are described in section 4 , Both base changing transformations and gauge transformations are described for the finite $q_{k}$ string states. Lifting these up to transformations on the Cauchy sequences gives transformations that take one real number representation to another, $R_{k, g} \rightarrow$ $R_{k^{\prime}, g^{\prime}}$.

The description is extended to include quantum theory representations of complex numbers, $C_{k, g}$, in section 5. They are defined as equivalence classes of

\footnotetext{
${ }^{2}$ An often used equivalent definition is based on Dedekind cuts of rational numbers instead of Cauchy sequences.
} 
Cauchy sequences of states of pairs of finite $q_{k}$ strings where the pair elements correspond to real and imaginary parts of a complex rational number. Cauchy conditions are applied separately to the sequences of real and imaginary components.

There is another very important difference between quantum and classical representations of real and complex numbers. This is the fact that the states of the $q_{k}$ strings used to define Cauchy sequences are elements of a Fock space that is itself a vector space over a field of real and complex numbers. For example all eigenvalues of operators acting on these string states are complex or real numbers. Also all linear superposition coefficients are complex numbers. This is quite different from the classical situation in that real and complex numbers play no role in the representation of numbers as states of bit or kit strings.

This dependence of quantum theory representations on the real and complex number base of spaces of $q_{k}$ string states leads to the possibility of iteration of the construction. Each representation $R_{k, g}, C_{k, g}$ can serve as the real and complex number base of Hilbert space and Fock space representations of $q_{k}$ string states that can be used to construct other representations of the real and complex numbers.

In addition, this same iteration possibility extends to all physical theories that are representable as mathematical structures over the real and complex numbers. Included are quantum and classical mechanics, quantum field theory, special and general relativity, string theory, as well as other theories.

This leads to the association of a reference frame $F_{k, g}$ to each representation $R_{k, g}, C_{k, g}$. Each frame $F_{k, g}$ contains representations of all physical theories as mathematical structures based on $R_{k, g}, C_{k, g}$. This use of reference frame terminology is consistent with other uses [27, 28] in that it sets a base or reference point $R_{k, U}, C_{k, U}$ for representations of all physical theories.

Much of the rest of the paper, Section [6 is concerned with properties of these reference frames and with three dimensional fields of these reference frames. Two of the dimensions are labeled by $k$ and $g$. The third is by an integer $j$ denoting the iteration stage. Different iteration possibilities are considered:, finite, one way infinite, two way infinite, and cyclic. Also properties of observers in different locations in the frame field are described.

Section 7 includes a discussion on what is probably the most important outstanding issue, how to integrate the frame field with physics. This is especially important from the viewpoint of constructing a coherent theory of physics and mathematics together [4, 5] or if one considers the physical universe as a mathematical universe [8, 9. Both relatively simple aspects of the possible integration, and more speculative aspects are described. However it is clear from this that much remains to be done to achieve an integration with physics.

The discussion section includes a description of the possible replacement of Cauchy sequences by operators, a possible use of gauge theory to integrate this work with physics, and other issues.

Two aspects of the following work should be emphasized. One is that rational numbers are represented by states of single qubit strings and not by states of pairs of qubit strings. This is based on the observation that all physical representations of rational numbers, such as computer inputs and outputs, outcomes of measure- 
ments, physical constants, etc. are as single strings of digits in some base $k \geq 2$ (usually 2 or 10) and not as integer pairs. Also complex numbers are represented in computations by pairs of finite digit strings in some base where the pair elements correspond to the real and imaginary components. The use of this representation is based on the fact that sets of rational numbers so represented are dense in the sets of all rational and all real numbers.

In this paper basic arithmetic relations and operations for the different types of numbers are discussed. The reason for this is based on the observation that states of kit or qukit strings, such as $|100101\rangle$ for $k=2$, do not, in any ab initio sense, represent numbers of any type. In order to show that these states represent numbers, one must prove that they satisfy a relevant set of axioms. The axioms are expressed in terms of properties of basic arithmetic relations and operations. It follows that a proof that sets of states of finite $q_{k}$ strings represent numbers is based on showing that definitions of these relations and operations satisfy the relevant axiom sets. Some details of these proofs, which are based on classical proofs, 25], are given in [6, 29].

\section{Quantum Representations of Natural Numbers, Integers, and Rational Numbers}

\section{$2.1 \quad$ Representations}

The quantum representations of numbers are described here by states of strings of qukits on a two dimensional integer lattice, $I \times I$. The states are given by $|\gamma, 0, h, s\rangle_{k, g}$ where $s$ is a $0,1, \cdots, k-1$ valued function on an interval $[l, h ; u, h]$ of $I \times I$, with $l \leq 0 \leq u, \gamma=+,-$ denotes the sign, and $0, h$ the lattice location of the $k-a l$ point. The reason for the subscript $g$ will be clarified later on.

Here it is intended that the states $|\gamma, 0, h, s\rangle_{k, g}$ represent numbers in $N, I$, and $R a$. For numbers in $N, \gamma=+, l=0$; for numbers in $I, l=0$, and there are no restrictions for $R a$. A compact notation is used where the location of the sign, denoted by $0, h$, is also the location of the $k-a l$ point. As examples, the base 10 numbers $612,-0474,-012.7100$ are represented here by $|612+\rangle,|0474-\rangle, \mid 012-$ $7100\rangle$ respectively. Note that leading and trailing $0 s$ are allowed.

The states $|\gamma, 0, h, s\rangle_{k, g}$ can be represented in terms of creation operators acting on the qukit vacuum state $|0\rangle$ where

$$
|\gamma, 0, h, s\rangle_{k}=c_{\gamma, 0, h}^{\dagger} a_{s(u, h), u, h}^{\dagger} \cdots a_{s(l, h), l, h}^{\dagger}|0\rangle=c_{\gamma, 0, h}^{\dagger}\left(a^{\dagger}\right)_{h}^{s}|0\rangle .
$$

Here $c_{\gamma, 0, h}^{\dagger}$ creates a sign qubit at $(0, h)$ and $a_{i, j, h}^{\dagger}$ creates a qukit in state $i=$ $0,1, \cdots, k-1$ at $(j, h) .\left(a^{\dagger}\right)_{h}^{s}$ is a short representation of the string of $a^{\dagger}$ operators.

The creation operators and the corresponding annihilation operators satisfy the usual commutation or anticommutation rules for respective boson or fermion qukits. The variable $h$ is present to allow for the presence of $n$-tuples of $q_{k}$ string states representing $n$-tuples of numbers.

The use of $I \times I$ as a framework for qukit state representations is based on the need to distinguish qukits in a string by a discrete ordering parameter and to 
distinguish different qukit strings from one another. This is seen in Eq. 11 where the integers $j$ with $l \leq j \leq u$ order the qukits in a string and the values of $h$ serve to distinguish different strings. There is no need to consider $I \times I$ as a lattice of points in a two dimensional physical space as its sole function is to provide discrete ordering and distinguishing labels.

Also the locations of the qukit strings in the lattice direction of the strings will be restricted in that the sign qubit will always be at site 0 . This restriction is inessential because the only function of the $j$ label in $(j, h)$ is to provide a discrete ordering of qukits states in a string.

The set of states $|\gamma, 0, h, s\rangle_{k, g}$ for all $\gamma, h, s$ are a basis, $\mathcal{B}_{k, h, g}$, that spans a Fock space $\mathcal{F}_{k, h}$ of states that are linear superpositions

$$
\psi=\sum_{\gamma, h, s} c_{\gamma, h, s}|\gamma, 0, h, s\rangle_{k, g}
$$

Here and in the following, $\sum_{s}=\sum_{l \leq 0} \sum_{u \geq 0} \sum_{s_{[l, u]}}$ is a sum over all integer intervals $[l, u]$ and over all $0,1, \cdots, k-1$ valued functions $s$ with domain $[l, u]$. A Fock space is used because states of $q_{k}$ strings with different numbers of qukits are included. The subscript $k \geq 2$ denotes the base. Note that base $k$ qukits are different from base $k^{\prime}$ qukits just as spin $k$ systems are different from spin $k^{\prime}$ systems.

Extension of the description to include pairs, triples and $n$-tuples of basis states and their linear superpositions is done by distinguishing different states in the tuples with different values of $h$. For each finite subset $S=h_{1}, h_{2}, \cdots, h_{|S|}$ of integers where $|S|$ is the number of integers in $S$, let $\mathcal{B}_{k, S, g}$ be the set of states of the form $\left|\gamma_{1}, 0, h_{1} s_{1}\right\rangle_{k}\left|\gamma_{2}, 0, h_{2}, s_{s}\right\rangle_{k} \cdots\left|\gamma_{|S|}, 0, h_{|S|}, s_{|S|}\right\rangle_{k}$. Define $\mathcal{B}_{k, g}$ by

$$
\mathcal{B}_{k, g}=\bigcup_{S} \mathcal{B}_{k, S, g}
$$

$\mathcal{B}_{k, g}$ is a basis set of all finite tuples of states of finite $q_{k}$ strings. Let $\mathcal{F}_{k}$ be the Fock space spanned by the states in $\mathcal{B}_{k, g}$.

The representation of state $n$-tuples used here is by products of states as in $\left|\gamma_{1}, 0, h_{1}, s_{1}\right\rangle_{k, g} \cdots\left|\gamma_{|S|}, 0, h_{|S|}, s_{|S|}\right\rangle_{k, g}$. The A-C operator representation of this state is $c_{\gamma_{1}, 0, h_{1}}^{\dagger}\left(a^{\dagger}\right)_{h_{1}}^{s_{1}} \cdots c_{\gamma_{|S|}, 0, h_{|S|}}^{\dagger}\left(a^{\dagger}\right)_{h_{|S|}}^{s_{|S|}}|0\rangle$. For bosons the ordering of the operators is immaterial. For fermions a specific ordering must be selected as a canonical ordering.

The basic arithmetic relations needed to show that the states $|\gamma, 0, h, s\rangle_{k, g}$ do represent numbers are equality $=_{A, k, g}$ and less than $<_{A, k, g} 3$

$$
|\gamma, 0, h, s\rangle_{k, g}={ }_{A, k, g}\left|\gamma^{\prime}, 0, h^{\prime}, s^{\prime}\right\rangle_{k, g}
$$

\footnotetext{
${ }^{3}$ One cannot avoid defining these relations and operations directly on the states. To see this let the operator $\tilde{N}$ satisfy $\tilde{N}|\gamma, 0, h, s\rangle_{k, g}=N(\gamma, s)|\gamma, 0, h, s\rangle_{k, g}$ where $N(\gamma, s)$ is supposed to be the number represented by $|\gamma, 0, h, s\rangle_{k, g}$. Because of the possible presence of leading and trailing $0 s$, the eigenspaces of $\tilde{N}$ are infinite dimensional. One knows that the set of eigenvalues of $\tilde{N}$ satisfy the relevant axioms. To prove that $N(\gamma, s)$ is the number represented by $|\gamma, 0, h, s\rangle_{k, g}$ one must show that $\tilde{N}$ is a homomorphism. This requires defining the arithmetic relations and operations directly on the states and showing that they satisfy the relevant axioms.
} 
holds if $\gamma^{\prime}=\gamma$ and $s^{\prime}=s$ up to leading and trailing $0 s 4$

Arithmetic ordering $<_{A, k, g}$ on $N$, and on positive $I$ and $R a$ states,

$$
|+, 0, h, s\rangle_{k, g}<_{A, k, g}\left|+, 0, h^{\prime}, s^{\prime}\right\rangle_{k, g}
$$

expresses the condition that the left hand state is arithmetically less than the right hand state 5 The extension to zero and negative $I$ and $R a$ states is given by the two conditions

$$
\begin{aligned}
& |+, 0, h, \overline{0}\rangle_{k, g,}<_{A, k, g}\left|+, 0, h^{\prime}, s^{\prime}\right\rangle_{k, g} \text { for all } s^{\prime} \neq \overline{0} \\
& |+, 0, h, s\rangle_{k, g}<_{A, k, g}\left|+, 0, h^{\prime}, s^{\prime}\right\rangle_{k, g} \\
& \rightarrow\left|-, 0, h^{\prime}, s^{\prime}\right\rangle_{k, g}<_{A, k, g}|-, 0, h, s\rangle_{k, g}
\end{aligned}
$$

Here $\overline{0}$ denotes a constant 0 sequence.

The $A$ subscript in these relations emphasizes that these are arithmetic relations on the states. They are quite different from the usual quantum mechanical relations between states. For instance, two states which differ by the number of leading or trailing $0 s$ are arithmetically equal but are not quantum mechanically equal.

The basic arithmetic operations on $R a$ are,,$+- \times$, and a set of division operations, $\div \ell$, one for each $\ell$. This expresses the fact that the set of $k$-ary rational string numbers is not closed under division when restricted to single finite length strings. However it is closed under division to any finite accuracy, $k^{-\ell}$. For each $k$, unitary operators for,,$+- \times$, and $\div_{\ell}$ are represented by $\tilde{+}_{A, k, g}, \tilde{-}_{A, k, g}$, $\tilde{\times}_{A, k, g}$, and $\stackrel{\sim}{\div}_{A, k, g, \ell}$. These operators, acting on pairs of $q_{k}$ string states as input, generate an output triple consisting of the pair of input states and a result string state.

To express this in a bit more detail, let $\tilde{O}_{A, k, g}$ represent any of the four operation types, $(O=+,-, \times, \div \ell$. $)$ Then

$$
\begin{aligned}
& \tilde{O}_{A, k, g}|\gamma, 0, h, s\rangle_{k, g}\left|\gamma^{\prime}, 0, h^{\prime}, s^{\prime}\right\rangle_{k, g} \\
& \quad=|\gamma, 0, h, s\rangle_{k, g}\left|\gamma^{\prime}, 0, h^{\prime}, s^{\prime}\right\rangle_{k, g}\left|\gamma^{\prime \prime}, 0, h^{\prime \prime}, s^{\prime \prime}\right\rangle_{k, g, O_{A}}
\end{aligned}
$$

The preservation of the input states is sufficient to ensure that the operators are unitary. The values of $h, h^{\prime}, h^{\prime \prime}$ are arbitrary except that they are all different.

In these equations the states $\left|\gamma^{\prime \prime}, 0, h^{\prime \prime}, s^{\prime \prime}\right\rangle_{k, g}$ with subscripts $O=+,-, \times, \div \ell$ give the results of the arithmetic operations. It is often useful to write them as

$$
\begin{aligned}
& \left|\gamma^{\prime \prime}, 0, h^{\prime \prime}, s^{\prime \prime}\right\rangle_{k, g,+}=\left|0, h^{\prime \prime},\left(\gamma^{\prime}, s^{\prime}+A \gamma, s\right)\right\rangle_{k, g}, \\
& \left|\gamma^{\prime \prime}, 0, h^{\prime \prime}, s^{\prime \prime}\right\rangle_{k, g,-}=\left|0, h^{\prime \prime},\left(\gamma^{\prime}, s^{\prime}-A \gamma, s\right)\right\rangle_{k, g}, \\
& \left|\gamma^{\prime \prime}, 0, h^{\prime \prime}, s^{\prime \prime}\right\rangle_{k, g, \times}=\left|0, h^{\prime \prime},\left(\gamma^{\prime}, s^{\prime} \times_{A} \gamma, s\right)\right\rangle_{k, g} \\
& \left|\gamma^{\prime \prime}, 0, h^{\prime \prime}, s^{\prime \prime}\right\rangle_{k, g, \div \ell}=\left|0, h^{\prime \prime},\left(\gamma^{\prime}, s^{\prime} \div A, \ell \gamma, s\right)\right\rangle_{k, g} .
\end{aligned}
$$

\footnotetext{
${ }^{4}$ That is, for all $j$, If $j$ is in both $[l, u]$ and $\left[l^{\prime}, u^{\prime}\right]$, then $s(j, h)=s^{\prime}\left(j, h^{\prime}\right)$. If $j$ is in $[l, u]$ and not in $\left[l^{\prime}, u^{\prime}\right]$, then $s(j, h)=0$. If $j$ is in $\left[l^{\prime}, u^{\prime}\right]$ and not in $[l, u]$, then $s^{\prime}\left(j, h^{\prime}\right)=0$. The domains of $s$ and $s^{\prime}$ are $[l, h ; u, h]$ and $\left[l^{\prime}, h^{\prime} ; u^{\prime}, h^{\prime}\right]$.

${ }^{5}$ The $<_{A, k}$ relation can be expressed by conditions on $s$ and $s^{\prime}$. Let $j_{\max }$ and $j_{\max }^{\prime}$ be the largest $j$ values such that $s\left(j_{\max }, h\right)>0$ and $s^{\prime}\left(j_{\text {max }}^{\prime}, h^{\prime}\right)>0$. Then $|+, 0, h, s\rangle_{k, g}<_{A, k, g}$ $\left|+, 0, h^{\prime}, s^{\prime}\right\rangle_{k, g}$ if $j_{\max }<j_{\max }^{\prime}$ or $j_{\max }=j_{\max }^{\prime}$ and $s\left(j_{\max , h}<s^{\prime}\left(j_{\max }^{\prime}, h^{\prime}\right)\right.$.
} 
The subscript $A$ on these operations distinguishes them as arithmetic operations. They are different from the quantum operations of linear superposition,,+- and product, $\times$ with no subscripts.

Extension of these operations to linear superposition states introduces entanglement. Use of Eq. 7 gives

$$
\begin{gathered}
\tilde{O}_{A, k, g} \psi \psi^{\prime}=\sum_{\gamma, h, s} \sum_{\gamma^{\prime}, h^{\prime}, s^{\prime} k, g}\langle\gamma, 0, h, s \mid \psi\rangle_{k, g}\left\langle\gamma^{\prime}, 0, h^{\prime}, s^{\prime} \mid \psi^{\prime}\right\rangle_{k, g} \\
\times|\gamma, 0, h, s\rangle_{k, g}\left|\gamma^{\prime}, 0, h^{\prime}, s^{\prime}\right\rangle_{k, g}\left|\gamma^{\prime \prime}, 0, h^{\prime \prime}, s^{\prime \prime}\right\rangle_{k, g, O_{A}} .
\end{gathered}
$$

Another operation that is essential for the axioms for $N$ and is useful for the others is that of the successor operation which corresponds to the +1 operation. For $q_{k}$ string states the definition can be expanded to include successor operators $\tilde{V}_{j}$ for each integer $j$. The action of $\tilde{V}_{j}$ on a base $k$ string state

$$
\tilde{V}_{j}|\gamma, 0, h, s\rangle_{k, g}=\left|\gamma^{\prime}, 0, h, s^{\prime}\right\rangle_{k, g}
$$

corresponds to the arithmetic addition of $k^{j}$ where $j$ is any integer. The usefulness of this operation is that the other arithmetic operations can be defined in terms of it.

Also this definition provides an efficient way 6 to implement the arithmetic operations [34. This follows from the observations that for each $k$

$$
\tilde{V}_{j}^{k}=\tilde{V}_{j+1}
$$

and that the implementation of each $\tilde{V}_{j}$ is efficient. Also implementation of the various arithmetic operations by use of the $\tilde{V}_{j}$ is efficient.

\subsection{Transformations of Representations}

As was noted earlier, the Fock space, $\mathcal{F}_{k}$, is spanned by the basis, $\mathcal{B}_{k, g}$, that is the set of all finite tuples of states of finite $q_{k}$ strings. Ultimately, $\mathcal{B}_{k, g}$ consists of sums and products of the individual $q_{k}$ bases, $\mathcal{B}_{k, h, j, g}$ where $\mathcal{B}_{k, h, j, g}$ is a set of $k$ single $q_{k}$ states that spans the $k$ dimensional Hilbert space $\mathcal{H}_{j, h}$ for site $j, h$.

As is well known there are an infinite number of choices for a basis set in a Hilbert space. Here $\mathcal{B}_{k, h, j, g}$ denotes one choice. A choice of a basis set for each $\mathcal{H}_{j, h}$ is equivalent to a gauge fixing. Thus a basis choice for each $j, h$ corresponds to a particular gauge choice at $j, h$. The subscript $g$ represents a a gauge fixing function where for each integer pair $j, h$,

$$
g(j, h)=\mathcal{B}_{k, j, h, g} .
$$

In what follows it is quite useful to treat $\mathcal{B}_{k, g}$ and $\mathcal{F}_{k}$ together. They will be denoted as $\mathcal{F B}_{k, g}$. One reason for this is that the arithmetic relations and operations, which are needed to prove that the states $|\gamma, 0, h, s\rangle_{k, g}$ represent numbers, in $N, I$, and $R a$, are defined on the states in $\mathcal{B}_{k, g}$ and extend by linearity to states in $\mathcal{F}_{k}$.

\footnotetext{
${ }^{6}$ The numbers of steps to implement the arithmetic operations are polynomial in the qukit string lengths.
} 
The arguments given so far show that the set of all $\mathcal{F} \mathcal{B}_{k, g}$ form a space parameterized by a space of 2 -tuples $(k, g)$. Here $k$ is a base and $g$ is a gauge fixing function defined by Eq. 12. Transformations $(k, g) \rightarrow\left(k^{\prime}, g^{\prime}\right)$ on the parameter space induce transformations $\mathcal{F} \mathcal{B}_{k, g} \rightarrow \mathcal{F B}_{k^{\prime}, g^{\prime}}$ on the representation space. The two transformations of interest are the $k$ changing transformations $\tilde{W}_{k^{\prime}, k}$ and the gauge transformations $U_{k}$. Gauge transformations of the sign qubit are ignored here although they could be easily included.

The gauge transformation, $U_{k}$ is a $U(k)=U(1) \times S U(k)$ valued function on $I \times I$. $U_{k}$ is global if $U_{k}(j, h)$ is independent of $j, h$. Otherwise it is local. The action of $U_{k}$ changes the basis set or state reference frame for each qukit [31] in that

$$
U_{k}(j, h) B_{k, j, h, g}=B_{k, j, h, g^{\prime}}
$$

holds for each $g$.

One can use the definition of $U_{k}$ to define gauge transformation operators on $\mathcal{B}_{k, h, g}$ and $\mathcal{B}_{k, g}$. Here notation will be abused in that $U_{k}$ will represent all these transformations. It will be clear from context which is meant.

The action of $U_{k}$ on a state $|\gamma, 0, h, s,\rangle_{k, g}$ and the individual A-C operators is given by

$$
\begin{aligned}
& |\gamma, 0, h, s\rangle_{k, g^{\prime}}=U_{k}|\gamma, 0, h, s\rangle_{k, g} \\
& \quad=c_{\gamma, 0, h}^{\dagger} U_{k}(u, h)\left(a_{k}^{\dagger}\right)_{s(u), u, h} \cdots U_{k}(l, h)\left(a_{k}^{\dagger}\right)_{s(l), l, h}|0\rangle \\
& \quad=c_{\gamma, 0, h}^{\dagger}\left(\left(a_{k}^{\dagger}\right)_{U_{k}(u, h)}\right)_{s(u), u, h} \cdots\left(\left(a_{k}^{\dagger}\right)_{U_{k}(l, h)}\right)_{s(l), l, h}|0\rangle
\end{aligned}
$$

where

$$
\begin{gathered}
\left(\left(a_{k}^{\dagger}\right)_{U_{k}(j, h)}\right)_{\alpha, j, h}=U_{k}(j, h)\left(a_{k}^{\dagger}\right)_{\alpha, j, h}=\sum_{\beta} U_{k}(j, h)_{\alpha, \beta}\left(a_{k}^{\dagger}\right)_{\beta, j, h} \\
\left(\left(a_{k}\right)_{U_{k}(j, h)}\right)_{\beta, j, h}=\left(a_{k}\right)_{\beta, j, h} U_{k}^{\dagger}(j, h)=\sum_{\alpha} U_{k}^{*}(j, h)_{\alpha, \beta} a_{\alpha, j, h}
\end{gathered}
$$

These results are based on the representation of $U_{k}(j, h)$ as

$$
U_{k}(j, h)=\sum_{\alpha, \beta}\left(U_{k}(j, h)\right)_{\alpha, \beta}\left(a_{k}^{\dagger}\right)_{\alpha, j, h}\left(a_{k}\right)_{\beta, j, h} .
$$

Here $\left(\left(a_{k}^{\dagger}\right)_{U_{k}(j, h)}\right)_{\alpha, j, h}$ is the creation operator for $q_{k}$ in the state $|\alpha, j, h\rangle_{k, g^{\prime}}$ in the basis $B_{k, j, h, g^{\prime}}$ just as $\left(a_{k}^{\dagger}\right)_{\alpha, j, h}$ is the creation operator for $q_{k}$ in the state $|\alpha, j, h\rangle_{k, g}$ in the basis $B_{k, j, h, g}$.

The base changing operator $\tilde{W}_{k^{\prime}, k}$ is more complex. If $\tilde{W}_{k^{\prime}, k}$ is defined on the state $|\gamma, 0, h, s\rangle_{k, g}$, then

$$
\left|\gamma, 0, h, s^{\prime}\right\rangle_{k^{\prime}, g}=\tilde{W}_{k^{\prime}, k}|\gamma, 0, h, s\rangle_{k, g}
$$

represents the same number in the base $k^{\prime}$ representation as $|\gamma, 0, h, s\rangle_{k}$ does in the base $k$ representation. This a nontrivial requirement because one needs to specify what is meant by "the same number as". In particular it means that all number theoretic properties are valid for $|\gamma, 0, h, s\rangle_{k}$, if and only if they are valid for $\left|\gamma, 0, h, s^{\prime}\right\rangle_{k^{\prime}}$. 
For any $k^{\prime}, k$, the operator $\tilde{W}_{k^{\prime}, k}$ is defined for all natural number and integer qukit string states. For qukit string states that represent rational numbers the domain and range of $\tilde{W}_{k^{\prime}, k}$ depend on the relations between the prime factors of $k$ and $k^{\prime}$. The domains and ranges for the different cases are summarized by the following relations [29]. Let $P F(k)$ denote the prime factors of $k$. Then

If $P F(k) \bigcap P F\left(k^{\prime}\right)=0$ then the domain and range of $\tilde{W}_{k^{\prime}, k}$ are the integer subspaces of $\mathcal{F} \mathcal{B}_{k, g}$ and $\mathcal{F} \mathcal{B}_{k^{\prime}, g}$

If $P F(k) \subset P F\left(k^{\prime}\right)$ then $\tilde{W}_{k^{\prime}, k} \mathcal{F} \mathcal{B}_{k, g} \subset \mathcal{F} \mathcal{B}_{k^{\prime}, g}$,

If $P F(k) \supset P F\left(k^{\prime}\right)$ then $\tilde{W}_{k^{\prime}, k} \subset \mathcal{F} \mathcal{B}_{k, g}=\mathcal{F} \mathcal{B}_{k^{\prime}, g}$,

If $P F(k), P F\left(k^{\prime}\right)$ each have elements not in the other and

share elements in common, then $\tilde{W}_{k^{\prime}, k} \subset \mathcal{F} \mathcal{B}_{k, g}=\subset \mathcal{F} \mathcal{B}_{k^{\prime}, g}$,

If $P F(k)=P F\left(k^{\prime}\right)$ then $\tilde{W}_{k^{\prime}, k} \mathcal{F} \mathcal{B}_{k, g}=\mathcal{F} \mathcal{B}_{k^{\prime}, g}$.

In the above $\subset \mathcal{F} \mathcal{B}_{k, g}$ denotes a subspace of $\mathcal{F} \mathcal{B}_{k, g}$ that contains the integer representations. In all these cases, if the state $|\gamma, 0, h, s\rangle_{k, g}$ is in the domain of $\tilde{W}_{k^{\prime}, k}$, then the base $k^{\prime}$ state, $\tilde{W}_{k^{\prime}, k}|\gamma, 0, h, s\rangle_{k, g}$, represents the same rational number as does $|\gamma, 0, h, s\rangle_{k, g}$.

The case where $P F(k)=P F\left(k^{\prime}\right)$ is of special interest because for each $k$ there is a smallest $k^{\prime}$ that has the same prime factors as $k$. If

$$
k=p_{j_{1}}^{h_{1}} \cdots p_{j_{n}}^{h_{n}}
$$

then the smallest $k^{\prime}$ is given by

$$
k^{\prime}=p_{j_{1}} \cdots p_{j_{n}} .
$$

Here $p_{j_{a}}$ for $a=1,2, \cdots, n$ is the $j_{a}$ th prime number. This shows that for each finite subset $S$ of primes, there is a set $\left[k_{S}\right]$ of bases such that for any pair $k, k^{\prime} \epsilon\left[k_{S}\right]$, $\tilde{W}_{k^{\prime}, k}$ is defined everywhere on $\mathcal{F} \mathcal{B}_{k, g}$ and $\tilde{W}_{k^{\prime}, k} \mathcal{F} \mathcal{B}_{k, g}=\mathcal{F} \mathcal{B}_{k^{\prime}, g}$.

A special case of this consists of the values $k_{n}$ whose factors are the first $n$ primes, each to the first power,

$$
k_{n}=p_{1} p_{2} \cdots p_{n}=2 \times 3 \times \cdots \times p_{n} .
$$

The sets $\left[k_{n}\right]$ are of interest here because, if $n<m$, then $\left[k_{n}\right] \subset\left[k_{m}\right]$. The limit properties, as $n \rightarrow \infty$, of $\left[k_{n}\right]$ and $\tilde{W}_{k^{\prime}, k_{n}}$ are open for investigation.

It should also be noted that the definitions of both $U_{k}$ and $\tilde{W}_{k^{\prime}, k}$ extend by linearity to linear superpositions of qukit string states. If $\psi=\sum_{\gamma, h, s} c_{\gamma, h, s}|\gamma, 0, h, s\rangle_{k, g}$, then

$$
\begin{aligned}
& U_{k} \psi=\sum_{\gamma, h, s} c_{\gamma, h, s} U_{k}|\gamma, 0, h, s\rangle_{k, g} \\
& \tilde{W}_{k^{\prime}, k} \psi=\sum_{\gamma, h, s} c_{\gamma, h, s} \tilde{W}_{k^{\prime}, k}|\gamma, 0, h, s\rangle_{k, g} .
\end{aligned}
$$

The validity of the second equation is restricted to the case where all component states with nonzero coefficients are in the domain of $\tilde{W}_{k^{\prime}, k}$.

It is of interest to note that there is in general no commutation relation between $U_{k}$ and $\tilde{W}_{k, k^{\prime}}$. The one exception is the case when $k^{\prime}=k^{n}$ for some $n$. 
However, for each pair $k, k^{\prime}$ for which $\tilde{W}_{k, k^{\prime}}$ is defined everywhere on $\mathcal{F}_{k}$, and for each pair $U_{k}, U_{k^{\prime}}^{\prime}$ one can define a transformed operator

$$
\left(\tilde{W}_{U^{\prime}, U}\right)_{k^{\prime}, k}=U_{k^{\prime}}^{\prime} \tilde{W}_{k^{\prime}, k} U_{k}^{\dagger} .
$$

This operator takes a transformed state $U_{k}|\gamma, h, s\rangle_{k, g}$ to a base $k^{\prime}$ state

$$
\left(\tilde{W}_{U^{\prime}, U}\right)_{k^{\prime}, k} U_{k}|\gamma, h, s\rangle_{k, g}=U_{k^{\prime}}^{\prime} \tilde{W}_{k^{\prime} . k}|\gamma, h, s\rangle_{k, g}
$$

that represents the same number in the $k^{\prime}, g^{\prime}$ representation as $|\gamma, h, s\rangle_{k, g}$ does in the $k, g$ representation. The steps in the representation transformations are

$$
(k, g) \stackrel{U_{k}}{\rightarrow}\left(k, g_{1}\right) \stackrel{\tilde{W}_{k_{k} k^{\prime}}}{\rightarrow}\left(k^{\prime}, g_{1}\right) \stackrel{U_{k^{\prime}}^{\prime}}{\rightarrow}\left(k^{\prime}, g^{\prime}\right) .
$$

Note that basis or gauge choice $g_{1}$ chosen for the base $k$ states is used to label the gauge choice for base $k^{\prime}$ states that are connected by $\tilde{W}_{k, k^{\prime}}$.

\subsection{Transformations of Arithmetic Relations and Opera- tions}

The arithmetic relations and operations transform in the expected way under the action of $\tilde{W}_{k, k^{\prime}}$ and $U_{k}$. One has

$$
\begin{gathered}
=_{A, k^{\prime}, g}=\left(\tilde{W}_{k, k^{\prime}}=_{A, k, g} \tilde{W}_{k, k^{\prime}}^{\dagger}\right) \\
\leq_{A, k^{\prime}, g}=\tilde{W}_{k, k^{\prime}} \leq_{A, k, g} \tilde{W}_{k, k^{\prime}}^{\dagger}
\end{gathered}
$$

for the relations and

$$
\begin{gathered}
O_{A, k^{\prime}, g}=\tilde{W}_{k, k^{\prime}} \times \tilde{W}_{k, k^{\prime}} \times \tilde{W}_{k, k^{\prime}} \\
\times O_{A, k, g} \tilde{W}_{k, k^{\prime}}^{\dagger} \times \tilde{W}_{k, k^{\prime}}^{\dagger}
\end{gathered}
$$

for $O=+, \times,-, \div \ell$.

These transformations of relations and operations hold without restrictions if and only if If $k$ and $k^{\prime}$ have the same prime factors. If this is not the case, then the restrictions expressed by Eq. 18 apply here. In the worst case where $k$ and $k^{\prime}$ are relatively prime, the transformations are restricted to the integer subspaces of $\mathcal{F}_{k}$ and $\mathcal{F}_{k^{\prime}}$. The presence of three transformation operators on the left of $O_{A, k, g}$ and two to the right accounts for the fact that $O_{A, k, g}$ preserves the two input strings and creates a third.

One has similar relations for the gauge transformations of relations and operations.

$$
\begin{gathered}
=_{A, k, g^{\prime}}=\left(U_{k}={ }_{A, k, g} U_{k}^{\dagger}\right) \\
\leq_{A, k^{\prime}, g}=U_{k} \leq_{A, k, g} U_{k}^{\dagger}
\end{gathered}
$$

for the relations and

$$
\begin{array}{r}
O_{A, k, g^{\prime}}=U_{k} \times U_{k} \times U_{k} \\
\times O_{A, k, g} U_{k}^{\dagger} \times U_{k}^{\dagger}
\end{array}
$$

for $O=+, \times,-, \div \ell$. 


\subsection{Unary Representations}

So far all number bases have been considered except one, the value $k=1$. The $k=1$ string representations are called unary representations. These are not usually considered, because basic arithmetic operations on these numbers are exponentially hard. For instance the number of steps needed to add two unary numbers is proportional to the values of the numbers and not to the logarithms of the values. However, even though they are not used arithmetically, they are always present in an interesting way.

To see this one notes that $k=1$ representations are the only ones that are extensive, all others are representational. The representational property for $k \geq 2$ base states of a qukit string means that a number represented by a state has nothing to do with the properties of the string state. The number represented by the state, $|672\rangle$, of a string of $3 q_{10}^{\prime} s$ is unrelated to the properties of the qukits in the state.

The extensiveness of a unary representation means that any collection of systems is an unary representation of a number that is the number of systems in the collection. There are many examples. A system of spins on a lattice is an unary representation of a number, that is the number of spins in the system. A gas of particles in a box is an unary representation of a number, that is the number of particles in the box. The qukit strings that play such an important role in this paper are unary representations of numbers, that are the number of qukits in the strings. A single qukit is an unary representation of the number 1.

The omnipresence of unary representations relates to another observation that 1 is the only number that is a common factor of all prime numbers and of all numbers. So it is present as a factor of any base. This ties in with the fact that unary representations of numbers are possible only for natural numbers and integers 7 Also there is the related observation that, for any pair $k, k^{\prime}$, the domain and range of $\tilde{W}_{k^{\prime}, k}$ include the integer subspaces of $\mathcal{F}_{k}$ and $\mathcal{F}_{k^{\prime}}$, and if $k, k^{\prime}$ have no prime factors in common, $\mathcal{F}_{k}$ and $\mathcal{F}_{k^{\prime}}$ are the domain and range of $\tilde{W}_{k^{\prime}, k}$.

The extensiveness of unary representations supports the inclusion of the $U(1)$ factor in the definition of $U_{k}$ as a $U(1) \times S U(k)$ valued function on $I \times I$. As a very simple example, a state $\left(a_{k}^{\dagger}\right)_{\alpha,(i, j)}|0\rangle$ of a qukit at location $(i, j)$ is an unary representation of the number 1 . Multiplication of this state by a phase factor $e^{i \theta_{i, j}}$ is a transformation that gives another state that is also an unary representation of the number 1 .

This argument extends to states of strings of qukits. A phase factor associated with any state of a string of $q_{k}$ at sites $(l, h), \cdots(u, h)$ is a product of the phase factors associated with each of the $q_{k}$ in the string. If $e^{i \theta_{j, h}}$ is a phase factor for

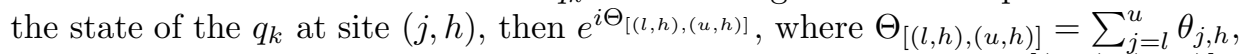
is the phase factor for the state of the $q_{k}$ string in the site interval $[(l, h),(u, h)]$.

As is well known, multiplying any state by a phase factor gives the same state as far as any physical meaning is concerned. However here one can have linear superpositions of states of strings of $q_{k}$ both at different locations and of different

\footnotetext{
${ }^{7}$ Non integer rational numbers require pairs of unary representations. However, pairs are not being considered here.
} 
length strings. In these cases the phase factors do matter to the extent that they can change the relative phase between the components in the superposition.

\subsection{Composite and Elementary Qukits}

So far the qukit components of strings are considered to be different systems for each value of $k$. A $k$ qukit is different from a $k^{\prime}$ qukit just as a spin $k$ system is different from a spin $k^{\prime}$ system. This leads to a large number of different qukit types, one for each value of $k$. However, the dependence of the properties of the base changing operator $\tilde{W}_{k^{\prime}, k}$ on the prime factors of $k$ and $k^{\prime}$ suggests that instead one consider qukits $q_{k}$ as composites $q_{c_{k}}$ of prime factor qukits $q_{p_{n}}$. In general the relation between the base $k q_{k}$ and the composite base $c(k) q_{c_{k}}$ is given by

$$
q_{c_{k}}=q_{p_{j_{1}}}^{h_{1}} q_{p_{j_{2}}}^{h_{2}} \cdots q_{p_{j_{n}}}^{h_{n}} .
$$

where (Eq. 19)

$$
k=p_{j_{1}}^{h_{1}} p_{j_{2}}^{h_{2}} \cdots p_{j_{n}}^{h_{n}}
$$

. Simple examples of this for $k=10$ and 18 are $q_{c_{10}}=q_{2} q_{5}$ and $q_{c_{18}}=q_{2} q_{3} q_{3}$.

The observation that for each $k$ there is a smallest $k^{\prime}$ with the same prime factors and its relevance to the properties of $\tilde{W}_{k^{\prime}, k}$ suggest the importance of the $q_{c_{k^{\prime}}}$ where the powers of the prime factors are all equal to 1(Eq. 200)

$$
q_{c_{k^{\prime}}}=q_{p_{j_{1}}} q_{p_{j_{2}}} \cdots q_{p_{j_{n}}} .
$$

A particular example of this for $k_{n}$, the product of the first $n$ prime numbers, is shown by (Eq. 21)

$$
q_{c_{k_{n}}}=q_{2} q_{3} q_{5} \cdots q_{p_{n}} .
$$

These considerations suggest a change of emphasis in that one should regard prime number qukits $q_{p_{n}}$ as basic or elementary and the qukits $q_{k}$ as composites of the elementary ones. In this case one would want to consider possible physical properties of the elementary qukits and how they interact and couple together to form composites. This is a subject for future work. It is, however, intriguing to note that if the prime number $q_{p_{n}}$ are considered as spin systems with spin $s_{n}$ given by $2 s_{n}+1=p_{n}$, then there is just one fermion, $q_{2}$. All the others are bosons.

As was the case for strings of $q_{k}$, one wants to represent numbers by states of finite strings of composite $q_{c_{k}}$. In general, this involves replacing the $k$ dimensional Hilbert space $\mathcal{H}_{k}$ at each site in $I \times I$ by a product space

$$
\mathcal{H}_{c_{k}}=\mathcal{H}_{p_{j_{1}}}^{h_{1}} \otimes \cdots \otimes \mathcal{H}_{p_{j_{n}}}^{h_{n}}
$$

and then following the development in the previous sections to describe number states. In particular the gauge fixing would apply to each component space in Eq. 32 for each location in $I \times I$.

The requirement that states of the form $\left|\gamma, 0, h, s^{\prime}\right\rangle_{c_{k^{\prime}}, g}$ represent numbers is based on an ordering of the basis states of $q_{c_{k}}$, or, what is equivalent, an ordering of the $n$-tuples in the range set of $s^{\prime}$. The definitions of arithmetic relations and 
operations for these states must respect the ordering and they must satisfy the relevant axioms and theorems for the type of number being considered.

The description of the transformation operations $\tilde{W}_{k^{\prime}, k}$ and $U_{k^{\prime}}$ can be extended to apply to the composite qukit strings. The base changing operator $\tilde{W}_{c_{k^{\prime}}, c_{k}}$ changes states of $q_{c_{k}}$ strings to states of $q_{c_{k^{\prime}}}$ strings that should represent the same number. Note that the expression of $\tilde{W}_{c_{k^{\prime}}, c_{k}}$ in terms of sums of products of AC operators will include the annihilation of many component elementary qukits in $q_{c_{k}}$ and creation of many that are components of $q_{c_{k^{\prime}}}$.

The description of gauge transformations $U_{c_{k}}$ applied to states of $q_{c_{k}}$ is interesting. If $q_{c_{k}}$ is composed of elementary $q_{p_{j}}$ as given by Eq. 30, then $U_{c_{k}}$ is a map from $I \times I$ to elements of $U\left(p_{j_{1}}\right) \times \cdots \times U\left(p_{j_{n}}\right)$. Here $U\left(p_{j_{i}}\right)$ is the unitary group of prime dimension $p_{j_{i}}$. For the special case of Eq. 31. $U_{c_{k_{n}}}$ takes values in $U\left(p_{1}\right) \times \cdots \times U\left(p_{n}\right)$. respectively. Since $U\left(p_{j}\right)=U(1) \times S U\left(p_{j}\right)$ the values of $U_{c_{k_{n}}}$ can be represented as elements of

$$
\begin{aligned}
U(1) \times & S U\left(p_{1}\right) \times S U\left(p_{2}\right) \times \cdots \times S U\left(p_{n}\right) \\
& =U(1) \times S U(2) \times S U(3) \times S U(5) \times \cdots \times S U\left(p_{n}\right) .
\end{aligned}
$$

Here the phase factor elements in $U(1)$ for each elementary qukit have been combined into one phase factor for the composite $q_{c_{k_{n}}}$.

This brief description of composite and elementary qukits shows that this may be an interesting approach to examine further. Problems to investigate include the nature of the coupling of elementary qukits to form a composite, invariance of properties of composite qukit string states under the action of $U_{k}$, particularly of $U_{c\left(k_{n}\right)}$, and other aspects.

The discussion so far suggests that, as far as quantum theory representations of natural numbers, integers, and rational numbers are concerned, it is sufficient to limit components of gauge transformations to products of elements of $U(1)$ and products of elements of $S U(p)$ groups where $p$ is a prime number. Furthermore it is sufficient that, for each prime $p$, elements of $S U(p)$ occur at most once in the product. It is also sufficient to limit components to products of the form of Eq. 33 for $n=1,2, \cdots$ as these will include representations for all rational numbers.

\section{Quantum Representations of Real Numbers}

Here quantum representations of real numbers are described as equivalence classes of sequences of base $k \geq 2$ qukit $\left(q_{k}\right)$ string states that satisfy the Cauchy condition 8 Sequences of states are defined to be functions $\Psi$ from the natural numbers to states in $\mathcal{F}_{k}$. If the states in the range set of $\Psi$ are all basis states in $\mathcal{B}_{k, g}$, then $\Psi(n)=\left|\gamma_{n}, h_{n}, s_{n}\right\rangle_{k, g}$. The values of $h_{n}$ in the states $\left|\gamma_{n}, h_{n}, s_{n}\right\rangle_{k}$ are all different in that $m \neq n \rightarrow h_{m} \neq h_{n}$. This is needed because one must be able to distinguish $\Psi(n)$ from $\Psi(m)$. Here and from now on the location 0 of the sign qubit in $|\gamma, 0, h, s\rangle_{k, g}$ is suppressed as it is always the same.

\footnotetext{
${ }^{8}$ This extends earlier work 6 on real number representations that was limited to $k=2$.
} 
These sequences extend classical representations in that the $\mathcal{B}_{k, g}$ valued sequences correspond to classical states of kit sequences. However sequences of linear superposition states have no classical correspondences.

\subsection{The Cauchy Condition for State Sequences}

The definition of the Cauchy condition for sequences of $q_{k}$ string states is a translation into quantum mechanics of a definition in mathematical analysis textbooks 26. To this end let $\Psi$ be a $\mathcal{B}_{k, g}$ valued sequence of $q_{k}$ string states. The sequence $\Psi$ satisfies the Cauchy condition if

For each $\ell$ there is a $p$ where for all $j, m>p$

$$
\left|\left(\left|\Psi(j)-{ }_{A, k, g} \Psi(m)\right|_{A, k, g}\right)\right\rangle_{k, g}<_{A, k, g}|+,-\ell\rangle_{k, g} .
$$

Here $\left|\left(\left|\Psi(j)-{ }_{A, k, g} \Psi(m)\right|_{A, k, g}\right)\right\rangle_{k, g}$ is the basis state that is the base $k$ arithmetic absolute value of the state resulting from the arithmetic subtraction of $\Psi(m)$ from $\Psi(j)$. The Cauchy condition says that this state is arithmetically less than or equal to the state $|+,-\ell\rangle_{k, g}=\left|+, 0_{[0,-\ell+1]} 1_{-\ell}\right\rangle_{k, g}$ for all $j, m$ greater than some $p$. Here $|+,-\ell\rangle$ represents the number $k^{-\ell}$. The subscripts $A, k, g$ are used to indicate that the operations are arithmetic and are defined for base $k$ string states in $\mathcal{B}_{k, g}$. They are not the usual quantum theory operations?

The Cauchy condition can be extended to sequences of linear superpositions of $q_{k}$ string states. Let $\Psi(n)=\sum_{\gamma, h, s}|\gamma, h, s\rangle_{k, g}\langle\gamma, h, s \mid \Psi(n)\rangle$. The probability that the arithmetic absolute value of the arithmetic difference between $\Psi(j)$ and $\Psi(m)$ is arithmetically less than or equal to $|+,-\ell\rangle$ is given by

$$
\begin{aligned}
P_{j, m, \ell}=\sum_{\gamma, h, s} \sum_{\gamma^{\prime}, h^{\prime}, s^{\prime}}\left|\langle\gamma, h, s \mid \Psi(j)\rangle\left\langle\gamma^{\prime}, h^{\prime}, s^{\prime} \mid \Psi(m)\right\rangle\right|^{2}: & \\
\mid(\mid \gamma, h, s-A, k, g & \left.\left.\gamma^{\prime}, h^{\prime},\left.s^{\prime}\right|_{A, k, g}\right)\right\rangle_{k, g} \leq_{A, k, g}|+,-\ell\rangle_{k, g} .
\end{aligned}
$$

The sum is over all $|\gamma, h, s\rangle,\left|\gamma^{\prime}, h^{\prime}, s^{\prime}\right\rangle$ that satisfy the statement in the second line of the above equation.

The definition of the probability $P^{\Psi}$ that $\Psi$ satisfies the Cauchy condition is obtained from the values of $P_{n, m, \ell}^{\Psi}$ by taking account of the quantifiers in the definition in Eq. 34, To this end define the probabilities $P_{p, \ell}^{\Psi}, P_{\ell}^{\Psi}$, and $P^{\Psi}$ by

$$
\begin{gathered}
P_{p, \ell}^{\Psi}=\inf _{n, m>p} P_{n, m, \ell}^{\Psi} \\
P_{\ell}^{\Psi}=\lim _{\sup _{p \rightarrow \infty}} P_{p, \ell}^{\Psi}=\lim _{p \rightarrow \infty} P_{p, \ell}^{\Psi} \\
P_{C}^{\Psi}=\liminf _{\ell \rightarrow \infty} P_{\ell}^{\Psi}=\lim _{\ell \rightarrow \infty} P_{\ell}^{\Psi} .
\end{gathered}
$$

This definition is based on the structure of the Cauchy condition in Eq. 34. It shows that the asymptotic values of $P_{n, m, \ell}^{\Psi}$ as $m, n \rightarrow \infty$ are important. The values for any particular $m, n$ or finite set $\{m, n\}$ of values (with $\ell$ fixed) for each $\ell$ are not important. The structure also shows that $P_{p, \ell}^{\Psi}$ is a non decreasing function of $p$ and that $P_{\ell}^{\Psi}$ is a non increasing function of $\ell$.

\footnotetext{
${ }^{9}$ When it is desired to emphasize the dependence of the definition of the Cauchy condition on $g$, Eq. 34 will be referred to as the g-Cauchy condition.
} 
The sequence $\Psi$ is said to be a Cauchy sequence if $P_{\Psi}$ is equal to 1 . A necessary and sufficient condition for this to occur is that $P_{n, m, \ell}^{\Psi} \rightarrow 1$ as $n, m \rightarrow \infty$ for each $\ell$. That is,

Theorem $1 P_{\Psi}=1 \Leftrightarrow \lim _{m, n \rightarrow \infty} P_{n, m, \ell}^{\Psi}=1$ for each $\ell$.

Proof sufficiency: Obvious as probabilities are bounded above by 1 , One has $\overline{P_{p, \ell}^{\Psi}}=1$ for each $p$ and $\ell$. This gives $P_{\ell}^{\Psi}=1$ for each $\ell$.

necessity: Assume $P_{\Psi}=q<1$. From the definition of $P_{\ell}^{\Psi}$ one sees that it approaches $q$ from above as $\ell$ increases. It follows that for sufficiently large $\ell, P_{p, \ell}^{\Psi}$, which is non decreasing is bounded from above by $q$ as $p$ increases. It follows from the definition of $P_{p, \ell}^{\Psi}$ that either $\lim _{m, n \rightarrow \infty} P_{n, m, \ell}^{\Psi}$ does not exist or it exists and is $\leq q$. QED

There are many examples of sequences $\Psi$ that are Cauchy with probability 1. A simple example is the following: Let $s$ be a $0,1, \cdots, k-1$ valued function on the non positive integers $[0,-\infty]$. Define $\Psi(n)$ by

$$
\Psi(n)=\left|+, 0, h, s_{[0,-n+1]}\right\rangle_{k} \times \frac{1}{\sqrt{k}} \sum_{j=0}^{k-1}|-n, h, j\rangle_{k} .
$$

Here $|j, h,-n\rangle_{k}$ denotes a $q_{k}$ at site $-n, h$ in state $j$.

The sequence $\Psi$ is Cauchy with probability 1 because $P_{n, m, \ell}^{\Psi}=1$ for all $m, n>\ell$. Also this example does not correspond to any classical Cauchy sequence of rational numbers. Additional examples are given in [6].

\subsection{Basic Relations on State Sequences}

One way to proceed is to define the field relations and operations on equivalence classes of sequences and show that these satisfy the real number axioms. However this method does not make clear the relation between the basic arithmetic relations on the $q_{k}$ string states and those on the equivalence classes. The method used here is to define the basic relations and operations on the sequences in terms of the relations and operations on the $q_{k}$ string states and use them to define the equivalence classes and field relations and operations on the classes.

As a piece of nomenclature let $R e_{i, S, k, g}$ denote the two relations where $R e_{i, S, k, g}$ is $=_{S, k, g}$ (equality) for $i=1$ and $R e_{i, S, k, g}$ is $<_{S, k, g}$ (less than) for $i=2$. The simplest definition one thinks of is an elementwise definition:

$$
\Psi(n) R e_{i, S, k, g} \Psi^{\prime} \Leftrightarrow \forall n \Psi R e_{i, A, k, g} \Psi^{\prime}(n) .
$$

Here $R e_{i, A, k, g}$ corresponds to the two relations on finite $q_{k}$ string states.

These definitions are unsatisfactory in that they are too strong. For $i=1$ this definition gives the result that for most $\mathcal{F}_{k}$ valued sequences, $\Psi$, the probability that $\Psi=_{S, k, g} \Psi$ is 0 . This holds even if $\Psi$ is Cauchy. For $i=2$ the definition of $<_{S, k, g}$ does not have the right asymptotic properties. 
A better definition of $=_{S, k, g}$ is an asymptotic definition. Let $\Psi$ and $\Psi^{\prime}$ be $\mathcal{B}_{k, g}$ valued sequences. Then

$$
\begin{aligned}
& \Psi=\infty, k, g \Psi^{\prime} \Leftrightarrow \\
& \forall \ell \exists p \forall j, m>p\left|\left(\left|\Psi(j)-{ }_{A, k, g} \Psi^{\prime}(m)\right|_{A, k, g}\right)\right\rangle_{k}<_{A, k, g}|+,-\ell\rangle_{k, g} .
\end{aligned}
$$

This definition is the same as the Cauchy condition of Eq. 34 except that $\Psi^{\prime}(m)$ replaces $\Psi(m)$. This definition says nothing about whether specific elements of $\Psi$ equal the corresponding ones of $\Psi^{\prime}$. It says that the elements of $\Psi$ and $\Psi^{\prime}$ must approach each other asymptotically. It is easy to show that this definition satisfies the requirement for a definition of equality. It is reflexive, symmetric, and transitive.

An asymptotic definition of ordering is given by

$$
\begin{aligned}
& \Psi<\infty, k, g \Psi^{\prime} \Leftrightarrow \\
& \quad \exists \ell \exists p \forall j, m>p\left|\Psi^{\prime}(j)-{ }_{A, k, g} \Psi(m)\right\rangle_{k}>_{A, k, g}|+,-\ell\rangle_{k} .
\end{aligned}
$$

This is also an asymptotic definition in that it says that $\Psi$ is less than $\Psi^{\prime}$ if $\Psi$ is asymptotically arithmetically less than $\Psi^{\prime}$ by some fixed amount, $|+,-\ell\rangle_{k}$. This definition differs from Eq. 39 in that $\forall \ell$ is replaced by $\exists \ell$, there is no arithmetic absolute value, and $<_{A, k, g}$ is replaced by $>_{A, k, g}$.

These definitions can be extended to $\mathcal{F}_{j, k}$ valued sequences. Let $\Psi$ and $\Psi^{\prime}$ be sequences of this type. Define $P_{n, m, \ell}^{\Psi=\infty} \Psi^{\prime}$ by,

$$
\begin{aligned}
& P_{n, m, \ell}^{\Psi=\infty} \Psi^{\prime}=\sum_{\gamma, h, s} \sum_{\gamma^{\prime}, h^{\prime}, s^{\prime}}\left|d_{\gamma, h, s}^{n} f_{\gamma^{\prime}, h^{\prime}, s^{\prime}}^{m}\right|^{2}: \\
& \left|\left(\left|(\gamma, h, s)-{ }_{A, k, g}\left(\gamma^{\prime}, h^{\prime}, s^{\prime}\right)\right|_{A, k, g}\right)\right\rangle_{k} \leq_{A, k, g}|+,-\ell\rangle_{k}
\end{aligned}
$$

where

$$
\begin{gathered}
d_{\gamma, h, s}^{n}=\langle\gamma, h, s \mid \Psi(n)\rangle \\
f_{\gamma^{\prime}, h^{\prime}, s^{\prime}}^{m}=\left\langle\gamma^{\prime}, h^{\prime}, s \mid \Psi^{\prime}(m)\right\rangle .
\end{gathered}
$$

Here $P_{n, m, \ell}^{\Psi=\infty} \Psi^{\prime}$ is the probability that $\Psi(n)$ and $\Psi^{\prime}(m)$ satisfy the relation in the second line of Eq. 41

Let $P^{\Psi=\infty} \Psi^{\prime}$ be the probability that $\Psi={ }_{\infty} \Psi^{\prime}$, i. e. that $\Psi$ equals $\Psi^{\prime} S$ asymptotically. Here $P^{\Psi=\infty} \Psi^{\prime}$ is given by

$$
\begin{gathered}
P_{p, \ell}^{\Psi=\infty \Psi^{\prime}}=\inf _{n, m>p} P_{n, m, \ell}^{\Psi=\infty} \Psi^{\prime} \\
P_{\ell=\infty}^{\Psi=\Psi^{\prime}}={\lim \sup _{p \rightarrow \infty} P_{p, \ell}^{\Psi=\infty} \Psi^{\prime}=\lim _{p \rightarrow \infty} P_{p, \ell}^{\Psi=\infty} \Psi^{\prime}}^{P^{\Psi=\infty} \Psi^{\prime}=}=\liminf _{\ell \rightarrow \infty} P_{\ell}^{\Psi=\infty} \Psi^{\prime}=\lim _{\ell \rightarrow \infty} P_{\ell}^{\Psi=\infty} \Psi^{\prime} .
\end{gathered}
$$

These equations are similar to those in Eq. 36] because the quantifier setup in Eq. 39 is the same as that for the Cauchy condition in Eq. 34. As was the case before, $P_{p, \ell}^{\Psi=\Psi^{\prime}}$ is a non decreasing function of $p$ for each $\ell$ and $P_{\ell}^{\Psi=\Psi^{\prime}}$ is a non increasing function of $\ell$.

A similar result holds for the probability $P^{\Psi<\infty} \Psi^{\prime}$ that $\Psi$ is asymptotically less than $\Psi^{\prime}$. Eqs. 40 and 42 give

$$
\begin{aligned}
& P_{n, m, \ell}^{\Psi<\infty} \Psi^{\prime}=\sum_{\gamma, h, s} \sum_{\gamma^{\prime}, h^{\prime}, s^{\prime}}\left|d_{\gamma, h, s}^{n} f_{\gamma^{\prime}, h^{\prime}, s^{\prime}}^{m}\right|^{2}: \\
& \quad\left|\left(\gamma^{\prime}, h^{\prime}, s^{\prime}\right)-A, k, g(\gamma, h, s)\right\rangle_{k, g} \geq_{A, k, g}|+,-\ell\rangle_{k, g} .
\end{aligned}
$$


From Eq. 40 one obtains results for $P^{\Psi<\infty} \Psi^{\prime}$ that are different from Eq. 43

$$
\begin{aligned}
& P_{p, \ell}^{\Psi<\infty} \Psi^{\prime}=\inf _{n, m>p} P_{n, m, \ell}^{\Psi<\infty} \Psi^{\prime} \\
& P_{\ell}^{\Psi<\infty} \Psi^{\prime}=\limsup _{p \rightarrow \infty} P_{p, \ell}^{\Psi<\infty \Psi^{\prime}}=\lim _{p \rightarrow \infty} P_{p, \ell}^{\Psi<\infty \Psi^{\prime}} \\
& P_{\Psi<\infty} \Psi^{\prime}=\lim \sup _{\ell \rightarrow \infty} P_{\ell}^{\Psi<\infty}=\lim _{\ell \rightarrow \infty} P_{\ell}^{\Psi<\infty} \Psi^{\prime} .
\end{aligned}
$$

These definitions have some satisfying properties. One is that if $\Psi$ and $\Psi^{\prime}$ are Cauchy sequences then exactly one of the following relations is true with probability 1 and the other two are false (true with probability 0 ):

$$
\begin{aligned}
& \Psi=_{\infty, k, g} \Psi^{\prime} \\
& \Psi>_{\infty, k, g} \Psi^{\prime} \\
& \Psi>_{\infty, k, g} \Psi^{\prime}
\end{aligned}
$$

This result follows from the observation that 0,1 are the only possible values for $P_{\Psi R e_{i, \infty, k, g} \Psi^{\prime}}$ for $\mathcal{F}_{k}$ valued sequences, $\Psi, \Psi^{\prime}$, provided that $\Psi$ and $\Psi^{\prime}$ are both Cauchy. That is

$$
\Psi \text { and } \Psi^{\prime} \text { are Cauchy } \Rightarrow P_{\Psi e_{i, \infty, k, g} \Psi^{\prime}}=0 \text { or } 1 .
$$

To see this it is sufficient to examine $=_{\infty, k, g}$ as the proofs for the other two relations are similar. One can rewrite Eq. 41 in the equivalent form

$$
P_{n, m, \ell}^{\Psi=\infty} \Psi^{\prime}=\sum_{\gamma, h, s} \sum_{\gamma^{\prime}, h^{\prime}, s^{\prime}}^{\prime}\left|d_{\gamma, h, s}^{n}\right|^{2}\left|f_{\gamma^{\prime}, h^{\prime}, s^{\prime}}^{m}\right|^{2}
$$

The prime on the $\gamma^{\prime}, h^{\prime}, s^{\prime}$ sum mean that the sum is restricted to states $\left|\gamma^{\prime}, h^{\prime}, s^{\prime}\right\rangle$ that are at least as large as $\left|(g, h, s)-{ }_{A}(+,-\ell)\right\rangle$ and no larger than $\mid(g, h, s)+{ }_{A}$ $(+,-\ell)\rangle$. Since the states $\Psi$ and $\Psi^{\prime}$ are Cauchy, the distributions $\left|d_{\gamma, h, s}^{n}\right|^{2}$ and $\left|f_{\gamma^{\prime}, h^{\prime}, s^{\prime}}^{m}\right|^{2}$ become increasingly narrow as $n, m$ increase.

The distributions either lie on top of one another for each $\ell$ or they do not. In the first case, for sufficiently large $m, n$ the restrictions on the $\gamma^{\prime}, h^{\prime}, s^{\prime}$ sum can be ignored and $\lim _{m, n \rightarrow \infty} P_{n, m, \ell}^{\Psi=\infty} \Psi^{\prime}=1$ for all $\ell$. In the second case there is some $\ell$ for which the state $|+,-\ell\rangle$ approximately separates the distributions. For this and all larger $\ell$ values, the overlap probability $P_{n, m, \ell}^{\Psi=\infty} \Psi^{\prime}$ in Eq. 48 approaches 0 as $m, n \rightarrow \infty$.

Another useful property of the asymptotic relation $=_{\infty}$ is that for each $\mathcal{F}_{k}$ valued Cauchy sequence $\Psi$ there is a $\mathcal{B}_{k, g}$ valued sequence $\Psi^{\prime}$ such that $P^{\Psi=\infty} \Psi^{\prime}=$ 1 and $\Psi^{\prime}$ is Cauchy. The definition of $\Psi^{\prime}$ and proof that $\Psi^{\prime}$ is Cauchy and are summarized here. The proof that $P^{\Psi==_{\infty} \Psi^{\prime}}=1$, or that $\Psi={ }_{\infty} \Psi^{\prime}$, will not be given as it is similar to that for the Cauchy property of $\Psi^{\prime}$.

Define $\Psi^{\prime}(n)=\left|\gamma_{n}, h_{n}, s_{n}\right\rangle_{k, g}$ to be the state that maximizes the probability $P_{n, l}^{\Psi}\left(\gamma^{\prime}, h^{\prime}, s^{\prime}\right)$ where

$$
\begin{aligned}
& P_{n, l}^{\Psi}\left(\gamma^{\prime}, h^{\prime}, s^{\prime}\right)=\sum_{\gamma, h, s}\left|d_{\gamma, h, s}^{n}\right|^{2}: \\
& \quad \|(\gamma, h, s)-A, k, g \\
& \left.\left.\quad\left(\gamma^{\prime}, h^{\prime}, s^{\prime}\right)\right|_{A, k, g}\right\rangle_{k} \leq_{A, k, g}|+, \ell\rangle_{k, g} .
\end{aligned}
$$


Define $Q_{n, \ell}^{\Psi}$ to be that maximum:

$$
Q_{n, \ell}^{\Psi}=P_{n, l}^{\Psi}\left(\gamma_{n}, h_{n}, s_{n}\right)
$$

Since $Q_{n, \ell}^{\Psi} \geq P_{n, l}^{\Psi}\left(\gamma^{\prime}, h^{\prime}, s^{\prime}\right)$, multiplying both sides by $\left|d_{\gamma^{\prime}, h^{\prime}, s^{\prime}}^{m}\right|^{2}$ and carrying out the $\operatorname{sum} \sum_{\gamma^{\prime}, h^{\prime}, s^{\prime}}$ gives

$$
Q_{n, \ell}^{\Psi} \geq P_{n, m, l}^{\Psi}
$$

Since $\Psi$ is Cauchy,

$$
\lim _{n \rightarrow \infty} Q_{n, \ell}^{\Psi}=1 .
$$

To show that $\Psi^{\prime}$ is Cauchy, it is sufficient to prove that $\lim _{m, n \rightarrow \infty} W_{m, n, \ell}=0$ where

$$
\begin{aligned}
& W_{m, n, \ell}=\sum_{\gamma, h, s} \sum_{\gamma^{\prime}, h^{\prime}, s^{\prime}}\left|d_{\gamma, h, s}^{n}\right|^{2}\left|d_{\gamma^{\prime}, h^{\prime}, s^{\prime}}^{m}\right|^{2}: \\
& \left.\quad \|\left(\gamma_{n}, h_{n}, s_{n}\right)-\left.{ }_{A, k, g}\left(\gamma_{m}, h_{m}, s_{m}\right)\right|_{A, k, g}\right\rangle>_{A, k, g}|+,-3 \ell\rangle_{k, g} .
\end{aligned}
$$

If the condition is true, then $\lim _{m, n \rightarrow \infty} W_{m, n, \ell}=1$; if it is false, then $\lim _{m, n \rightarrow \infty} W_{m, n, \ell}=$ 0. Also $|+,-3 \ell\rangle_{k, g}=|+,-\ell\rangle_{k, g}+{ }_{A}|+,-\ell\rangle_{k, g}+_{A}|+,-\ell\rangle_{k, g}$.

Define $X_{m, n, \ell}$ by

$$
\begin{aligned}
& X_{m, n, \ell}=\sum_{\gamma, h, s} \sum_{\gamma^{\prime}, h^{\prime}, s^{\prime}}\left|d_{\gamma, h, s}^{n}\right|^{2}\left|d_{\gamma^{\prime}, h^{\prime}, s^{\prime}}^{m}\right|^{2}: \\
& \left.\left.\|\left(\gamma_{n}, h_{n}, s_{n}\right)-\left.A_{A}(\gamma, h, s)\right|_{A}\right\rangle++_{A} \|(\gamma, h, s)-\left._{A}\left(\gamma^{\prime}, h^{\prime}, s^{\prime}\right)\right|_{A}\right\rangle \\
& \left.\quad+_{A} \|\left(\gamma^{\prime}, h^{\prime}, s^{\prime}\right)-\left.{ }_{A}\left(\gamma_{m}, h_{m}, s_{m}\right)\right|_{A}\right\rangle>_{A}|+,-3 \ell\rangle_{k, g} .
\end{aligned}
$$

(Subscripts $k, g$ are suppressed here.) Since

$$
\begin{gathered}
\left.\|\left(\gamma_{n}, h_{n}, s_{n}\right)-\left.{ }_{A}\left(\gamma_{m}, h_{m}, g_{m}\right)\right|_{A}\right\rangle \\
\left.\left.\leq_{A} \|\left(\gamma_{n}, h_{n}, s_{n}\right)-\left.{ }_{A}(\gamma, h, s)\right|_{A}\right\rangle+_{A} \|(\gamma, h, s)-\left.{ }_{A}\left(\gamma^{\prime}, h^{\prime}, s^{\prime}\right)\right|_{A}\right\rangle, \\
\left.\quad+{ }_{A} \|\left(\gamma^{\prime}, h^{\prime}, s^{\prime}\right)-\left.{ }_{A}\left(\gamma_{m}, h_{m}, s_{m}\right)\right|_{A}\right\rangle
\end{gathered}
$$

one has the result that

$$
W_{m, n, \ell} \leq X_{m, n, \ell} .
$$

The condition in the definition of $X_{m, n, \ell}$ is satisfied only if at least one of the component states is $\geq_{A}|+,-\ell\rangle$. If this holds for the first or third component, then $X_{m, n, \ell} \leq 1-Q_{n, \ell}$ or $X_{m, n, \ell} \leq 1-Q_{m, \ell}$. If it holds for the second component, then $X_{m, n, \ell} \leq 1-P_{m, \ell}^{\Psi}$.

Eq. 52 and the fact that $\Psi$ is Cauchy gives the result that $\lim _{m, n \rightarrow \infty} X_{m, n, \ell}=$ 0. It follows from Eq. 53 that $\lim _{m, n \rightarrow \infty} W_{m, n, \ell}=0$. This gives the final result that $\left.\left|\left(\gamma_{m}, h_{m}, s_{m}\right)-_{A}\left(\gamma_{n}, h_{n}, s_{n}\right)\right|_{A}\right\rangle \leq_{A}|+,-\ell\rangle$ and thus that $\Psi^{\prime}$ is Cauchy.

\subsection{Basic Operations on State Sequences}

The problems requiring the definition of asymptotic relations do not appear to be present in the definition of basic relations on the sequences. For $\mathcal{B}_{k, g}$ valued sequences $\Psi$ and $\Psi^{\prime}$ one uses Eqs. 7 and 8 to define $O_{\nu, S, k, g}$ by

$$
O_{\nu, S, k, g} \Psi \Psi^{\prime}=\Theta
$$


where for $\nu=1,2,3$ and each $n$,

$$
\Theta(n)=\Psi(n) \times \Psi^{\prime}(n) \times \Psi^{\prime \prime}(n)
$$

and

$$
\Psi^{\prime \prime}(n)=\Psi(n) O_{\nu, A, k, g} \Psi^{\prime}(n) .
$$

The product structure of the elements of $\Theta$ allows one to write

$$
\Theta=\Psi \Psi^{\prime} \Psi^{\prime \prime}
$$

as the product of 3 state sequences.

For $\nu=4$ one division operator, $\div$ S,k,g , can be defined as an operator that is diagonal in the infinite number of state division operators, $\div{ }_{A, \ell}$. One has

$$
{ }_{S, k, g} \Psi \Psi^{\prime}=\Psi \Psi^{\prime} \Psi^{\prime \prime}
$$

where

$$
\Psi^{\prime \prime}(n)=\Psi(n) \div{ }_{A, k, g, n} \Psi^{\prime}(n) .
$$

Note the subscript $n$ in $\div{ }_{A, k, g, n}$.

Application of this definition to more general $\mathcal{F}_{k}$ valued sequences $\Psi$ and $\Psi^{\prime}$ generates a single sequence $\Theta$ of entangled states that cannot be represented in the product form of Eq. 57. From Eqs. 9 and 42, one has

$$
O_{\nu, A, k, g} \Psi \Psi^{\prime}=\Theta
$$

where

$$
\begin{aligned}
& \Theta(n)=\sum_{\gamma, h, s} \sum_{\gamma^{\prime}, h^{\prime}, s^{\prime}}\left|d_{\gamma, h, s}^{n}\right|^{2}\left|f_{\gamma^{\prime}, h^{\prime}, s^{\prime}}^{n}\right|^{2} \\
& |\gamma, h, s\rangle\left|\gamma^{\prime}, h^{\prime}, s^{\prime}\right\rangle\left|(\gamma, h, s) O_{\nu, A, k, g}\left(\gamma^{\prime}, h^{\prime}, s^{\prime}\right)\right\rangle .
\end{aligned}
$$

As shown, $\Theta$ is not a result sequence in the sense that $\Psi^{\prime \prime}$ was. To obtain a result sequence one must take the trace over the two initial states for each element of $\Theta$. This gives a sequence $\mathcal{P}_{\Psi, \Psi}$ of density operators where

$$
\begin{aligned}
& \mathcal{P}_{\Psi, \Psi}(n)=\sum_{\gamma, h, s} \sum_{\gamma^{\prime}, h^{\prime}, s^{\prime}}\left|d_{\gamma, h, s}^{n}\right|^{2}\left|f_{\gamma^{\prime}, h^{\prime}, s^{\prime}}^{n}\right|^{2} \\
& \quad \times \rho_{\left|(\gamma, h, s) O_{\nu, A, k, g}\left(\gamma^{\prime}, h^{\prime}, s^{\prime}\right)\right\rangle .}
\end{aligned}
$$

Inclusion of these sequences into the definitions presented so far requires expansion of the material to define the Cauchy condition and asymptotic relations for sequences of density operators. Since this has not yet been done, this will be left to future work. Also it is not clear if element definitions of $O_{\nu, S, k, g}$, as is done in Eq. 55, are useful here. In any case, one can proceed without these extensions. Also the main results are not affected by this lack.

\subsection{Quantum Representation of Real Numbers}

The asymptotic equality relation $=_{\infty, k, g}$ can be used to define equivalence classes of Cauchy sequences. Two sequences $\Psi$ and $\Psi^{\prime}$ are equivalent if and only if they are asymptotically equal:

$$
\Psi \equiv \Psi^{\prime} \Leftrightarrow \Psi=_{\infty, k, g} \Psi^{\prime}
$$


It is straightforward to show from the properties of $=_{\infty, k, g}$ that $\equiv$ has the right properties for a definition of equivalence.

For each Cauchy sequence $\Psi$ let $[\Psi]$ denote the equivalence class containing $\Psi$. As might be guessed, the set of all these equivalence classes is a quantum representation of the real numbers. Let $R_{k, g}$ denote the set. The subscripts $k, g$ indicate that the representation depends on both the base $k$ and the gauge or basis choice $g$.

As has been seen each class $[\Psi]$ contains many $\mathcal{F}_{k}$ valued sequences and at least one $\mathcal{B}_{k, g}$ valued sequence. From this one concludes that the quantum equivalence classes are larger than the classical equivalence classes but that no new classes are present.

The basic relations and operations can be lifted from sequences to the equivalence classes to define the basic relations and operations for a real number field. Let $R e_{i, R, k, g}$ denote the two relations $=_{R, k, g}$ for $i=1$ and $<_{R, k, g}$ for $i=2$. Let $[\Psi]$ and $\left[\Psi^{\prime}\right]$ denote two equivalence classes of Cauchy sequences. Then

$$
[\Psi] R e_{i, R, k, g}\left[\Psi^{\prime}\right] \Leftrightarrow \Psi R e_{i, \infty, k, g} \Psi^{\prime} .
$$

This definition holds for all $\mathcal{F}_{k}$ valued sequences.

The field operations, $O_{\nu, R, k, g}$ for $\nu=1-4(+, \times,-, \div)$, can be defined on equivalence classes through their definitions on $\mathcal{B}_{k, g}$ valued sequences. Let $\Psi, \Psi^{\prime}, \Psi^{\prime \prime}$ be Cauchy sequences that satisfy Eqs. 54 and 57 Define $O_{\nu, R, k, g}$ by

$$
[\Psi] O_{\nu, R, k, g}\left[\Psi^{\prime}\right]=\left[\Psi^{\prime \prime}\right] .
$$

This use of $\mathcal{B}_{k, g}$ valued sequences to define the field operations definition is done only because Cauchy sequences of density operators are not included here. Their inclusion would allow direct definitions of the field operators for all Cauchy sequences.

To verify that $R_{k, g}$ is a representation of the real numbers, one must show that $R_{k, g}$ and the relations, $R e_{i, R, k, g}$, and operations $O_{\nu, R, k, g}$, satisfy the real number axioms of a complete ordered field [25. Some details of this were given in [6, so it will not be repeated here. The proof is, in many ways, similar to that given for the usual classical Cauchy sequences of rational numbers [26].

\section{Space of Real Number Representations and As- sociated Transformations}

As described, the quantum theory representations of real numbers, $R_{k, g}$, depend on a base $k$ and a gauge $g$. Recall that $k$ denotes the dimensionality of the Hilbert space of states for each single $q_{k}$ and $g$ denotes a gauge field of basis sets on $I \times I$, Eq. 12 .

For each pair $k, g$ one has a quantum representation $R_{k, g}$ of the real numbers. Any pair, $R_{k, g}, R_{k^{\prime}, g^{\prime}}$ of real number representations are isomorphic as all representations of the real numbers (axiomatized by second order axioms) are isomorphic [35. However this does not mean that they are identical. For instance, 
Cauchy sequences of $q_{k}$ string states, which are elements of the equivalence classes in $R_{k, g}$, are distinct from Cauchy sequences of $q_{k^{\prime}}$ string states, which are elements of equivalence classes in $R_{k^{\prime}, g}$, as $q_{k}$ and $q_{k^{\prime}}$ systems are different.

Similarly $\mathcal{B}_{k, g}$ valued sequences $\Psi$ of $q_{k}$ string states are different from $\mathcal{B}_{k, g^{\prime}}$ valued sequences. Also the definition of the Cauchy condition, Eq. 34 is both $k$ and $g$ dependent. These dependencies can be seen from Eqs. 13]16] which show the relations between the two basis sets and between the single $q_{k} \mathrm{~A}-\mathrm{C}$ operators for each of the two basis sets.

These considerations show that the set of all representations $R_{k, g}$ can be regarded as a space of representations parameterized by a two dimensional space of all pairs, $k, g$. Transformations $k, g \rightarrow k^{\prime}, g^{\prime}$ induce transformations $R_{k, g} \rightarrow$ $R_{k^{\prime}, g^{\prime}}$ on the representation space. The components of the transformations on the representation space are operators that change bases, $R_{k, g} \rightarrow R_{k^{\prime}, g}$, and operators that change the gauge, $R_{k, g} \rightarrow R_{k, g^{\prime}}$.

\subsection{Gauge Changing Operators}

Gauge changing operators that act on sequences can be defined from the gauge transformations, $U_{k}$ as defined in Eqs. 13 and Eq. 14. To achieve this, let $\Psi$ and $\Psi^{\prime}$ be respective $\mathcal{B}_{k, g}$ and $\mathcal{B}_{k, g^{\prime}}$ valued sequences where

$$
\begin{gathered}
\Psi(n)=\left|\gamma_{n}, h_{n}, s_{n}\right\rangle_{k, g} \\
\Psi^{\prime}(n)=\left|\gamma_{n}, h_{n}, s_{n}\right\rangle_{k, g^{\prime}} .
\end{gathered}
$$

Define the operator, $\mathcal{U}_{k}$, by

$$
\Psi^{\prime}=\mathcal{U}_{k} \Psi
$$

where

$$
\left|\gamma_{n}, h_{n}, s_{n}\right\rangle_{k, g^{\prime}}=U_{k}\left|\gamma_{n}, h_{n}, s_{n}\right\rangle_{k, g}
$$

Here $g$ and $g^{\prime}$ are related by

$$
g^{\prime}=U_{k} g
$$

This shows that the elements of $\Psi^{\prime}, \Psi^{\prime}(n)$, are the same states, relative to the transformed basis as the elements,$\Psi(n)$, of $\Psi$ are, relative to the original basis. However, relative to the original basis, the states $\Psi^{\prime}(n)$ are different from the states $\Psi(n)$. This can be seen by expanding the states $\Psi^{\prime}(n)$ in terms of the original basis.

The definition of $\mathcal{U}_{k}$ extends by linearity to $\mathcal{F}_{k}$ valued sequences. If $\Psi$ is such a sequence where

$$
\Psi(n)=\sum_{\gamma, h, s} d_{\gamma, h, s}^{n}|\gamma, h, s\rangle_{k, g}
$$

then $\Psi^{\prime}$ is related to $\Psi$ by Eq. 67 where

$$
\Psi^{\prime}(n)=\sum_{\gamma, h, s} d_{\gamma, h, s}^{n}|\gamma, h, s\rangle_{k, g^{\prime}}
$$

Note the replacement of $g$ by $g^{\prime}$ on the right hand side. 
The definition of $\mathcal{U}_{k}$ can be lifted to apply to equivalence classes of Cauchy sequences to relate $R_{k, g}$ to $R_{k, g^{\prime}}$ as in $R_{k, g^{\prime}}=\mathcal{U}_{k} R_{k, g}$. The validity of this depends on the preservation of the Cauchy property under the action of $U_{k}$. That is, if $\Psi$ is a g-Cauchy sequence, then $\Psi^{\prime}=\mathcal{U}_{k} \Psi$ is a $g^{\prime}$-Cauchy sequence 10 To show that this is the case one has to define the g'-Cauchy condition relative to the basis states in $\mathcal{B}_{k, g}$. This is

$$
\begin{gathered}
\forall \ell \exists p \forall j, m>p\left|\left(\left|U_{k}\left(\gamma_{j}, h_{j}, s_{j}\right)-{ }_{A, k, g^{\prime}} U_{k}\left(\gamma_{m}, h_{m}, s_{m}\right)\right|_{A, k, g^{\prime}}\right)\right\rangle_{k, g^{\prime}} \\
\leq{ }_{A, k, g^{\prime}} U_{k}|+,-\ell\rangle_{k, g} .
\end{gathered}
$$

Here $U_{k}\left|\gamma_{j}, h_{j}, s_{j}\right\rangle_{k, g}=\left|\gamma_{j}, h_{j}, s_{j}\right\rangle_{k, g^{\prime}}, \leq_{A, k, g^{\prime}}=U_{k} \leq_{A, k, g} U_{k}^{\dagger}$, and $-_{A, k, g^{\prime}}=$ $U_{k} \times U_{k} \times U_{k}-{ }_{A, k, g} U_{k}^{\dagger} \times U_{k}^{\dagger}$. It is a straightforward exercise to show that, for this definition, the Cauchy property is preserved under the action of $U_{k}$.

The definition of $\mathcal{U}_{k}$ shows that these operators form a group of transformations. If $\mathcal{U}_{k}$ and $\mathcal{U}_{k}^{\prime}$ are gauge transformations, for Cauchy sequences, or for equivalence classes in $R_{k, g}$, then so is their product $\mathcal{U}_{k} \mathcal{U}_{k}^{\prime}$. Also each $\mathcal{U}_{k}$ has an inverse $\mathcal{U}_{k}^{-1}$. The group property follows from the fact that the $U_{k}$, on which the $\mathcal{U}_{k}$ are based, are products of elements of the unitary group $U(k)$.

\subsection{Base Changing Operators}

One would like to describe the base changing transformations for Cauchy sequences by lifting the base changing transformations $\tilde{W}_{k^{\prime}, k}$ for the $q_{k}$ string states to transformations on the Cauchy sequences. One first thinks of doing this by defining an operator $\mathcal{W}_{k^{\prime}, k}$ on $\mathcal{B}_{k, g}$ valued sequences $\Psi$. One would set

$$
\Psi^{\prime}=\mathcal{W}_{k^{\prime}, k} \Psi
$$

Here $\Psi^{\prime}$ is a $\mathcal{B}_{k^{\prime}, g}$ valued sequence such that for each $n$

$$
\Psi^{\prime}(n)=\left|\gamma_{n}, h_{n}, s_{n}^{\prime}\right\rangle_{k^{\prime}, g}=\tilde{W}_{k^{\prime}, k}\left|\gamma_{n}, h_{n}, s_{n}\right\rangle_{k, g}=\tilde{W}_{k^{\prime}, k} \Psi(n)
$$

The problem with this definition is that the domain and range of $\tilde{W}_{k^{\prime}, k}$ depend on the relation of the prime factors of $k$ and $k^{\prime}$. If $k$ and $k^{\prime}$ are relatively prime, then this definition fails as $\tilde{W}_{k^{\prime}, k}$ is not defined on any of the non integer states.

One way around this impasse is to generalize the definition of $\tilde{W}_{k^{\prime}, k}$ to operators $\tilde{W}_{k^{\prime}, k, \ell}$ for different nonnegative integers $\ell$. Here

$$
\left|\gamma_{n}, h_{n}, s_{n}^{\prime}\right\rangle_{k^{\prime}, g}=\tilde{W}_{k^{\prime}, k, \ell}\left|\gamma_{n}, h_{n}, s_{n}\right\rangle_{k, g}
$$

is a base $k^{\prime}$ state that represents the same number as the base $k$ state $\left|\gamma_{n}, h_{n}, s_{n}\right\rangle_{k, g}$ up to accuracy $|+,-\ell\rangle_{k^{\prime}, g}$. This removes the problem because, for each $\ell, \tilde{W}_{k^{\prime}, k, \ell}$ is defined on all $q_{k}$ string states in $\mathcal{F}_{k}$. Also $\tilde{W}_{k^{\prime}, k, \ell}=\tilde{W}_{k^{\prime}, k}$ on the integer state subspace of $\mathcal{F}_{k}$.

\footnotetext{
${ }^{10}$ The $g^{\prime}$-Cauchy condition is given by Eq. 34 with the subscript $g^{\prime}$ replacing $g$.
} 
The desired definition of $\mathcal{W}_{k^{\prime}, k}$ is that it be an isomorphism from $R_{k, g}$ to $R_{k^{\prime}, g}$. This is equivalent to requiring that $\mathcal{W}_{k^{\prime}, k} \Psi$ belongs to the equivalence class in $R_{k^{\prime}, g}$ that represents the same number as the equivalence class in $R_{k, g}$ that contains $\Psi$. A proposed method of achieving this is by a definition that is diagonal in $n$ and in $\ell$.

To this end one defines $\mathcal{W}_{k^{\prime}, k}$ by replacing $\tilde{W}_{k^{\prime}, k}$ with $\tilde{W}_{k^{\prime}, k, n}$ in Eq. 74 to get

$$
\Psi^{\prime}(n)=\left|\gamma_{n}, h_{n}, s_{n}^{\prime}\right\rangle_{k^{\prime}, g}=\tilde{W}_{k^{\prime}, k, n}\left|\gamma_{n}, h_{n}, s_{n}\right\rangle_{k, g}=\tilde{W}_{k^{\prime}, k, n} \Psi(n) .
$$

The operator $\mathcal{W}_{k^{\prime}, k}$ must satisfy two properties: The sequence $\Psi=\mathcal{W}_{k^{\prime}, k} \Psi$ must be Cauchy if $\Psi$ is Cauchy, and the two sequences, $\Psi^{\prime}$ in $R_{k^{\prime}, g}$ and $\Psi$ in $R_{k, g}$, must represent the same real number. (Here and in the following, Cauchy sequences will often be stand ins for equivalence classes of the sequences.) An equivalent requirement is that $\mathcal{W}_{k^{\prime}, k}$ is an isomorphism from $R_{k, g}$ to $R_{k^{\prime}, g}$. It preserves the basic field relations of equality and ordering and the operations of addition, multiplication and their inverses.

\section{Quantum Representations of Complex Numbers}

The simplest path to descriptions of quantum representations of complex numbers is their representation as ordered pairs of real number representations. If $[\Psi]$ and $\left[\Psi^{\prime}\right]$ represent two quantum real numbers, then $\left([\Psi],\left[\Psi^{\prime}\right]\right)$ represents a quantum complex number where $[\Psi]=[\Psi]^{r}$ and $\left[\Psi^{\prime}\right]=\left[\Psi^{\prime}\right]^{i}$ represent the real and imaginary components. The basic field relations $=_{R, k, g},<_{R, k, g}$ and operations $+_{R, k, g}, \times_{R, k, g},-{ }_{R, k, g}, \div R, k, g$ would be extended to relations $={ }_{C, k, g},<_{C, k, g}$ and operations $+_{C, k, g}, \times_{C, k, g},-C_{C, k, g}, \div_{C, k, g}$ following the standard rules.

The rest of this section can be skipped over by readers using the above definitions. The following development is based on the observation that all physical representations of complex numbers, such as in computations, are by ordered pairs of single string representations of rational numbers. This corresponds here to extending the treatment of rational number representations, as states of $q_{k}$ strings, to ordered pairs of states of $q_{k}$ strings. These represent the real and imaginary components of complex rational numbers. Application of the Cauchy condition separately to the real and imaginary components gives a description of Cauchy sequences of complex rational number representations. This gives quantum representations of complex numbers as equivalence classes of these Cauchy sequences.

One way to proceed is to continue working with one type of qukit but increase the number of sign qubit types from one to two 11 . The two qubit types are represented by A-C operators $c_{\gamma, 0, h}^{\dagger}, d_{\delta, 0, h}^{\dagger}$ and their complex conjugates. Here $c_{\gamma, 0, h}^{\dagger}$ and $d_{\delta, 0, h}^{\dagger}$ represent sign creation operators for the real and imaginary number components where $\gamma=+,-$ and $\delta=+i,-i$ at site $0, h^{\prime}$

Complex rational numbers are represented here by pairs of qukit string states, $\left|h, \gamma, s ; h^{\prime}, \delta, t\right\rangle_{k, g}$, with different $h$ values. In terms of A-C operators one has

$$
\left|h, \gamma, s ; h_{1}, \delta, t\right\rangle_{k, g}=c_{\gamma, 0, h}^{\dagger}\left(a^{\dagger}\right)_{[(l, h),(u, h)]}^{s} ; d_{\delta, 0, h_{1}}^{\dagger}\left(a^{\dagger}\right)_{\left[\left(l^{\prime}, h_{1}\right),\left(u^{\prime}, h_{1}\right)\right]}^{t}|0\rangle .
$$

\footnotetext{
${ }^{11}$ This differs from the approach in [6] which uses two types of qukits and qubits.
} 
The state $|0\rangle$ denotes the qukit vacuum and $0, h$ and $0, h_{1}$ denote the locations of the sign qubits. As before $[(l, h),(u, h)]$ and $\left[\left(l^{\prime}, h_{1}\right),\left(u^{\prime}, h_{1}\right)\right]$ denote lattice intervals where $l \leq 0 \leq u$ and $l^{\prime} \leq 0 \leq u^{\prime}$. Also

$$
\begin{gathered}
\left(a^{\dagger}\right)_{[(l, h),(u, h)]}^{s}=a_{s(u), u, h}^{\dagger} a_{s(u-1), u-1, h}^{\dagger} \cdots a_{s(l), l, h}^{\dagger} \\
\left(a^{\dagger}\right)_{\left[\left(l^{\prime}, h_{1}\right),\left(u^{\prime}, h_{1}\right)\right]}^{t}=a_{t\left(u^{\prime}\right), u^{\prime}, h_{1}}^{\dagger} a_{t\left(u^{\prime}-1\right), u^{\prime}-1, h_{1}}^{\dagger} \cdots a_{t\left(l^{\prime}\right),\left(l^{\prime}, h_{1}\right)}^{\dagger}
\end{gathered}
$$

where $s$ and $t$ are $0, \cdots, k-1$ valued functions with integer interval domains $[l, u]$ and $\left[l^{\prime}, u^{\prime}\right]$ respectively. The subscript $g$ denotes the implicit gauge choice for the $q_{k}^{r}$ string states at each site of $I \times I$.

A consequence of this representation is that if one has many pairs of string states, they are expressed in the A-C formalism as one long string of creation operators acting on $|0\rangle$. One then needs a method of determining the association between the imaginary and real strings. One of the different ways to do this is to describe the pairs as those in which $h$ is close or next to $h_{1}$. Here some method will be assumed implicitly as which one is used does not affect the results obtained in this paper.

The definitions of arithmetic relations and operations given for states of $q_{k}$ strings can be easily extended to states of pairs of $q_{k}$ strings following the usual arithmetic rules for operations on complex numbers. For arithmetic equality one has

$$
\begin{aligned}
& \left|h, \gamma, s ; h_{1}, \gamma_{1}, t\right\rangle_{k, g}=_{c, k, g}\left|h^{\prime}, \gamma^{\prime}, s^{\prime}, h_{1}^{\prime}, \gamma_{1}^{\prime}, t^{\prime}\right\rangle_{k, g} \\
& \Leftrightarrow\left(|h, \gamma, s\rangle_{k, g}={ }_{r, k, g}\left|h^{\prime}, \gamma^{\prime}, s^{\prime}\right\rangle_{k, g}\right. \\
& \text { and } \left.\left|h_{1}, \gamma_{1}, t\right\rangle_{k, g}={ }_{i, k, g}\left|h_{1}^{\prime}, \gamma_{1}^{\prime}, t^{\prime}\right\rangle_{k, g}\right) .
\end{aligned}
$$

Ordering relations are usually not considered because they are only partly defined (complex numbers cannot be ordered). The $c, r, i$ in the subscripts denote complex, real, and imaginary, respectively.

For the operations let $\tilde{O}_{c, k, g}$ be a unitary operator denoting any of the four operations $+_{c, k, g}, \times_{c, k, g},-_{c, k, g}, \div_{c, k, g, \ell}$. The action of any of these on complex rational states can be represented by

$$
\begin{aligned}
& \tilde{O}_{c, k, g}\left|h, \gamma, s ; h_{1}, \delta, t\right\rangle_{k, g}\left|h^{\prime}, \gamma^{\prime}, s^{\prime} ; h_{1}^{\prime}, \delta^{\prime}, t\right\rangle_{k, g} \\
& =\left|h, \gamma, s ; h_{1}, \delta, t\right\rangle_{k, g}\left|h^{\prime}, \gamma^{\prime}, s^{\prime} ; h_{1}^{\prime}, \delta^{\prime}, t^{\prime}\right\rangle_{k, g}\left|h^{\prime \prime}, \gamma^{\prime \prime},, s^{\prime \prime} ; h_{1}^{\prime \prime}, \delta^{\prime \prime}, t^{\prime \prime}\right\rangle_{k, g}
\end{aligned}
$$

where

$$
\left|h^{\prime \prime}, \gamma^{\prime \prime}, s^{\prime \prime} ; h_{1}^{\prime \prime}, \delta^{\prime \prime}, t^{\prime \prime}\right\rangle_{k, g}=_{c, k, g}\left|\left(h, \gamma, s ; h_{1}, \delta, t\right) O_{c, k, g}\left(h^{\prime}, \gamma^{\prime}, s^{\prime} ; h_{1}^{\prime}, \delta^{\prime}, t^{\prime}\right)\right\rangle_{k, g} .
$$

The expression $\left|\left(h, \gamma, s ; h_{1}, \delta, t\right) O_{c, k, g}\left(h^{\prime}, \gamma^{\prime}, s^{\prime} ; h_{1}^{\prime}, \delta^{\prime}, t^{\prime}\right)\right\rangle_{k, g}$ with $O$ inside $|-,-\rangle$ represents the rational string state resulting from carrying out the operation $O_{c, k, g}$. Unitarity is satisfied by preserving the two input states and creating a result state.

The arithmetic operations create entangled states when applied to linear superpositions of the basis states. One has

$$
\begin{gathered}
\tilde{O}_{c, k, g} \psi \psi^{\prime}=\sum_{h, \gamma, s, h_{1}, \delta, t} \sum_{h^{\prime}, \gamma^{\prime}, s^{\prime}, h_{1}^{\prime}, \delta^{\prime}, t^{\prime}} k, g_{\langle} h, \gamma, s ; h_{1}, \delta, t|\psi\rangle \\
\quad \times k, g\left\langle h^{\prime}, \gamma^{\prime}, s^{\prime} ; h_{1}^{\prime}, \delta^{\prime}, t^{\prime} \mid \psi^{\prime}\right\rangle\left|h, \gamma, s ; h_{1}, \delta, t\right\rangle_{k, g}\left|h^{\prime}, \gamma^{\prime}, s^{\prime} ; h_{1}^{\prime}, \delta^{\prime}, t^{\prime}\right\rangle_{k, g} \\
\times\left|\left(h, \gamma, s ; h_{1}, \delta, t\right) O_{c, k, g}\left(h^{\prime}, \gamma^{\prime}, s^{\prime} ; h_{1}^{\prime}, \delta^{\prime}, t^{\prime}\right)\right\rangle_{k, g}
\end{gathered}
$$


Taking the trace over the $\psi$ and $\psi^{\prime}$ component states gives a mixed state

$$
\begin{aligned}
& \rho_{\psi O_{c, k, g} \psi^{\prime}}=\sum_{h, \gamma, s, h_{1}, \delta, t} \sum_{h^{\prime}, \gamma^{\prime}, s^{\prime}, h_{1}^{\prime}, \delta^{\prime}, t^{\prime}}\left|\left\langle h, \gamma, s ; h_{1}, \delta, t \mid \psi\right\rangle\right|^{2} \\
& \times\left|\left\langle h^{\prime}, \gamma^{\prime}, s^{\prime} ; h_{1}^{\prime}, \delta^{\prime}, t^{\prime} \mid \psi^{\prime}\right\rangle\right|^{2} \rho_{\left(h, \gamma, s ; h_{1}, \delta, t\right) O_{c, k, g}\left(h^{\prime}, \gamma^{\prime}, s^{\prime} ; h_{1}^{\prime}, \delta^{\prime}, t^{\prime}\right)}
\end{aligned}
$$

that represents the result of the operation.

Determination of the exact form of the state $\left|h^{\prime \prime}, \gamma^{\prime \prime}, s^{\prime \prime} ; h_{1}^{\prime \prime}, \delta^{\prime \prime}, t^{\prime \prime}\right\rangle_{k, g}$ from Eq. 81 for the different arithmetic operations is somewhat lengthy, but straightforward. It involves translation of the usual rules for implementation of arithmetic operations on complex numbers into those on quantum states. For example, for multiplication one uses the relations

$$
\begin{gathered}
d_{\gamma, 0, h}^{\dagger} \times d_{\gamma^{\prime}, 0, h^{\prime}}^{\dagger}=c_{\gamma^{\prime \prime}, 0, h^{\prime \prime}}^{\dagger} \text { where } \gamma^{\prime \prime}=+,[-] \text { if } \gamma \neq[=] \gamma^{\prime} \\
c_{\gamma, 0, h}^{\dagger} \times d_{\gamma^{\prime}, 0, h^{\prime}}^{\dagger}=d_{\gamma^{\prime \prime}, 0, h^{\prime \prime}}^{\dagger} \text { where } \gamma^{\prime \prime}=\gamma^{\prime},\left[\gamma^{\prime} \neq \gamma^{\prime \prime}\right] \text { if } \gamma=+[\gamma=-] \\
c_{\gamma, 0, h}^{\dagger} \times c_{\gamma^{\prime}, 0, h^{\prime}}^{\dagger}=c_{\gamma^{\prime \prime}, 0, h^{\prime \prime}}^{\dagger} \text { where } \gamma^{\prime \prime}=+,[-] \text { if } \gamma=\gamma^{\prime}\left[\gamma \neq \gamma^{\prime}\right] .
\end{gathered}
$$

Quantum representations of complex numbers are based on application of the Cauchy condition to the real and imaginary components separately of a sequence of states of $q_{k}$ string pairs. The sequence $\Psi$ where $\Psi(n)=\left|h_{n}, \gamma_{n}, s_{n} ; h_{n}^{\prime}, \delta_{n}, t_{n}\right\rangle_{k, g}$ of states is a Cauchy sequence if the following is satisfied:

$$
\begin{aligned}
& \left.\forall \ell \exists p \forall j, m>p \|\left(h_{j}, \gamma_{j}, s_{j}\right)-\left._{r, k, g}\left(h_{m}, \gamma_{m}, s_{m}\right)\right|_{r, k, g}\right\rangle_{k, g}<_{r, k, g}|+,-\ell\rangle_{k, g} \\
& \text { and } \left.\|\left(h_{j}^{\prime}, \delta_{j}, t_{j}\right)-\left.{ }_{i, k, g}\left(h_{m}^{\prime}, \delta_{m}, t_{m}\right)\right|_{i, k, g}\right\rangle_{k, g}<_{r, k, g}|+,-\ell\rangle_{k, g} .
\end{aligned}
$$

Here $|+,-\ell\rangle_{k, g}$ is the state corresponding to the number $k^{-\ell}$.

Extension of the Cauchy condition to sequences of linear superpositions of complex rational string states is similar to that for sequences of superpositions of real rational states. Such a sequence is Cauchy if the probability is unity that both the real and imaginary components satisfy the Cauchy condition.

The definition of equivalence for the real number representations extends here to complex number representations. Two Cauchy sequences $\Psi$ and $\Psi^{\prime}$ are equivalent if the real and imaginary components of $\Psi$ and $\Psi^{\prime}$ are asymptotically equal. Let $\Psi$ and $\Psi^{\prime}$ be $\mathcal{B}_{k, g}$ valued sequences where for each $n$

$$
\begin{aligned}
\Psi(n) & =\left|h_{n}, \gamma_{n}, s_{n} ; h_{1, n}, \delta_{n}, t_{n}\right\rangle_{k, g} \\
\Psi^{\prime}(n) & =\left|h_{n}^{\prime}, \gamma_{n}^{\prime}, s_{n}^{\prime} ; h_{1, n}^{\prime}, \delta_{n}^{\prime}, t_{n}^{\prime}\right\rangle_{k, g} .
\end{aligned}
$$

Then

$$
\begin{gathered}
\Psi=\infty, S, k, g \\
=^{\prime} \text { if } \forall \ell \exists p \forall j, m>p \\
\left.\left.\|\left(h_{j}, \gamma_{j}, s_{j}\right)-\left.{ }_{r, k, g}\left(h_{m}^{\prime}, \gamma_{m}^{\prime}, s_{m}^{\prime}\right)\right|_{r, k, g}\right\rangle_{k, g} \leq_{r, k, g}|+,-\ell|\right\rangle_{k, g} \text { and } \\
\left.\left.\|\left(h_{1, j}, \delta_{j}, t_{j}\right)-\left.{ }_{i, k, g}\left(h_{1, m}^{\prime}, \delta_{m}^{\prime}, t_{m}^{\prime}\right)\right|_{i, k, g}\right\rangle_{k, g} \leq_{r, k, g}|+,-\ell|\right\rangle_{k, g} .
\end{gathered}
$$

From this definition one ha: 12

$$
\Psi \equiv \Psi^{\prime} \text { if } \Psi={ }_{\infty, S, k, g} \Psi^{\prime} .
$$

\footnotetext{
${ }^{12}$ It is easy to see that this definition of $\equiv$ has the necessary properties of symmetry, reflexivity, and transitivity. These follow from the corresponding properties of $=_{\infty, S, k, g}$.
} 
The set $C_{k, g}$ of complex numbers is defined to be the set of equivalence classes $[\Psi]$ where $\Psi$ is a Cauchy sequence of $q_{2}^{r} q_{k}, q_{2}^{i} q_{k}$ string pairs. Here $q_{2}^{r}$ and $q_{2}^{i}$ denote the real and imaginary sign qubits. As was the case for $R_{k, g}$, each equivalence class is larger than the corresponding classical equivalence class, but there are no new equivalence classes. This follows from the observation that each class contains at least one $\mathcal{B}_{k, g}$ valued sequence.

The basic field relation $=_{C, k, g}$ is defined by

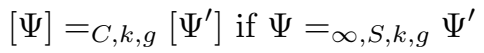

The operations $\tilde{+}_{C, k, g}, \tilde{-}_{C, k, g}, \tilde{x}_{C, k, g}, \div_{C, k, g}$, are defined in a similar fashion. For $\mathcal{B}_{k, g}$ valued Cauchy sequences one has expressions similar to Eqs. 54 et seq:

$$
\tilde{O}_{\nu, C, k, g}[\Psi]\left[\Psi^{\prime}\right]=[\Psi]\left[\Psi^{\prime}\right]\left[\Psi^{\prime \prime}\right]
$$

Here $\tilde{O}_{\nu, C, k, g}$ with $\nu=1,2,3,4$ is a stand in for the four operations. For $\nu=1,2,3$ the class $\left[\Psi^{\prime \prime}\right]$ contains all Cauchy sequences asymptotically equal to $\Psi^{\prime \prime}$ where

$$
\Psi^{\prime \prime}(n)=\left|\Psi(n) O_{\nu, c, k, g} \Psi^{\prime}(n)\right\rangle_{k, g} .
$$

For $\nu=4,\left(\div_{C, k, g}\right)$ one has a diagonal definition similar to Eq. $\left[5 \mathbb{f r}^{3}\right.$ :

$$
\Psi^{\prime \prime}(n)=\left|\Psi(n) \dot{c}_{c, k, g, n} \Psi^{\prime}(n)\right\rangle_{k, g} .
$$

As is the case for real number representations, these relations and operations extend to $\mathcal{F}_{k}$ valued Cauchy sequences. Details will not be given here as they are an extension of those for the real number representations.

\section{Fields of Quantum Reference Frames}

At this point it is good to step back and view some consequences of the existence of the many different representations of $R$ and $C$. All physical theories considered to date, and many mathematical theories, can be regarded as theories that are based on the real and complex numbers. Included are quantum and classical mechanics, quantum field theory, QED, QCD, special and general relativity, and string theory. It follows that for each representation $R_{k, g}, C_{k, g}$ of $R$ and $C$ one has a corresponding representation of physical theories as mathematical structures based on $R_{k, g}, C_{k, g}$

\footnotetext{
${ }^{13}$ The specific definitions of these operations follows those for complex numbers. As examples, for multiplication, if $\Psi(n)=\left|h_{n}, \gamma_{n}, s_{n} ; h_{1, n}, \delta_{n}, t_{n}\right\rangle_{k, g}=|x, i y\rangle$ and $\Psi^{\prime}(n)=$ $\left|h_{n}^{\prime}, \gamma_{n}^{\prime}, s_{n}^{\prime} ; h_{1, n}^{\prime}, \delta_{n}^{\prime}, t_{n}^{\prime}\right\rangle_{k, g}=\left|x^{\prime}, i y^{\prime}\right\rangle$, then

$$
\begin{array}{r}
\Psi^{\prime \prime}(n)=\mid\left(x \times_{r, k, g} x^{\prime}\right)-{ }_{r, k, g}\left(y \times_{i, k, g} y^{\prime}\right) ; \\
\left.\left(x \times_{i, k, g} y^{\prime}\right)+_{i, k, g}\left(x^{\prime} \times_{i, k, g} y\right)\right\rangle_{k, g} .
\end{array}
$$

Division to accuracy $n$ of $|x, y\rangle$ by $\left|x^{\prime}, y^{\prime}\right\rangle$ is done by carrying out the division to accuracy $n$ indicated by

$$
\left|x^{\prime \prime}, y^{\prime \prime}\right\rangle=\left|[\operatorname{Re}, \operatorname{Im}] \div{ }_{c, k, g, n}\left(x^{\prime} \times x^{\prime}\right)+\left(y^{\prime} \times y^{\prime}\right)\right\rangle
$$

where $R e=x \times x^{\prime}+y \times y^{\prime}$ and $\operatorname{Im}=x^{\prime} \times y-x \times y^{\prime}$.
} 
The large number of theories based on $R, C$ suggests that one associate a reference frame $F_{k, g}$ with each $R, C$ representation, $R_{k, g}, C_{k, g}$. Here $R_{k, g}, C_{k, g}$ is referred to as the base of frame $F_{k, g}$. The frame $F_{k, g}$ contains representations of all physical theories that are representable as structures based on $R_{k, g}, C_{k, g}$.

The large number of real and complex number representations and associated reference frames suggests that one define a frame field $F$ over the two dimensional parameter space $\{k, g\}$. The components of $F$, as a map from $\{k, g\}$ to a set of reference frames, are the frames $F_{k, g}$ at each value $k, g$. Note that the parameter $g$ is unique to quantum theory representations as it is not applicable to representations based on states of classical kit strings. However, the parameter $k$ is common to both qukit and kit string representations.

This construction is shown schematically in Figure 1 for three of the infinite number of values of $k, g$. This is shown by solid arrows coming from the parent frame $F_{R, C}$ to three of the infinitely many descendent frames.

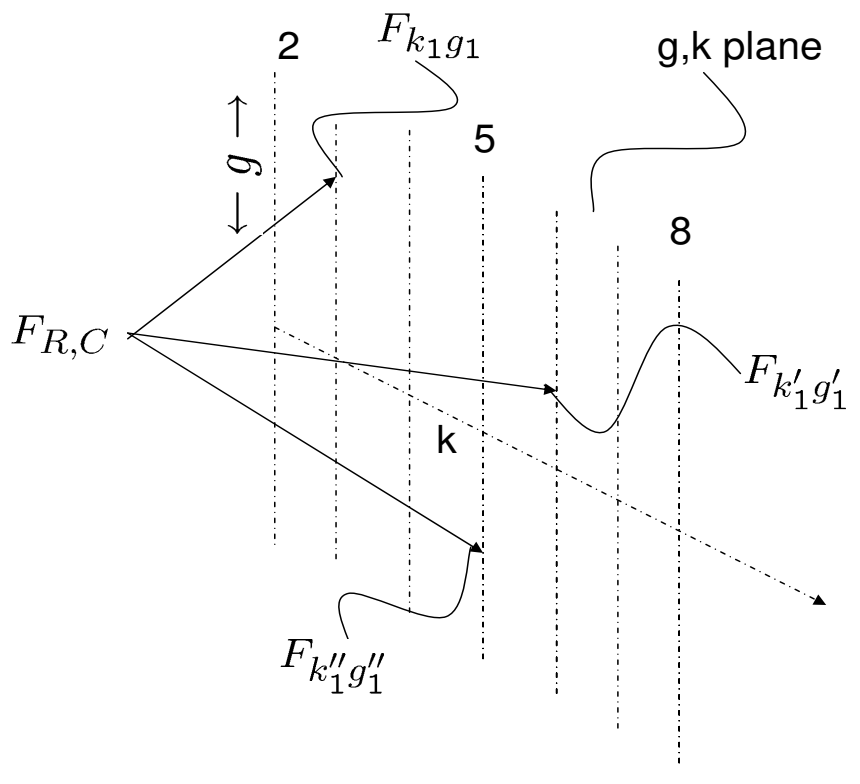

Figure 1: Schematic illustration of frames coming from frame $F_{R, C}$. The frames are based on quantum representations of real and complex numbers in $F_{R, C}$. The distinct vertical lines in the $k, g$ plane denote the discreteness of the integral values of $k \geq 2$. Only three of the infinitely many frames coming from $F_{R, C}$ are shown. Here $k$ denotes the qukit base and $g$ denotes a gauge or basis choice.

This use of reference frames has much in common with other uses of reference frames in physics and particularly in quantum theory [27, 28, 30, 31, 32, 33. In special relativity, inertial coordinate systems define reference frames for describing physical dynamics. In quantum cryptography, Alice and Bob pick a polarization direction to define a reference frame for sending messages encoded in qubit string states. Here each reference frame carries representations of all physical theories as mathematical structures based on the real and complex number base of the frame. 
It is of interest to examine what observers can and cannot see in the different frames. To begin it is assumed that an observer $O_{R}$ in the parent frame $F_{R, C}$ regards the real and complex numbers in the frame base as elementary objects. The only relevant properties they have are those required by the relevant axioms for $R$ and $d^{14}$. This assumption is based on the prevalent view taken by physics so far of the nature of real and complex numbers, that they are elementary objects. The only properties of these objects that physics cares about are those derivable from the relevant axioms.

The quantum theory representations of real and complex numbers described here suggest that $O_{R}$ sees that $R_{k, g}$ and $C_{k, g}$, as equivalence classes of Cauchy sequence of states of $q_{k}$ strings, represent real and complex numbers. $O_{R}$ also sees that $R_{k, g}$ and $C_{k, g}$, can serve as the base of a frame $F_{k, g}$ containing representations of physical theories as mathematical structures based on $R_{k, g}, C_{k, g}$.

Symmetry considerations suggest that an observer $O_{k, g}$, in each frame, $F_{k, g}$, has the same view relative to $F_{k, g}$ as $O_{R}$ does relative to the frame $F_{R, C}$. Thus $O_{k, g}$ sees $R_{k, g}, C_{k, g}$ as elementary, structures whose only relevant properties are those derivable from the relevant axiom sets. The structure of the elements of $R_{k, g}, C_{k, g}$, as equivalence classes of Cauchy sequences, seen by $O_{R}$, are not visible to $O_{k, g}$. Also the construction, in $F_{R, C}$, of representations, $R_{k, g}, C_{k, g}$, can be repeated in $F_{k, g}$ to obtain representations $R_{2, k^{\prime}, g^{\prime}}, C_{2, k^{\prime}, g^{\prime}}$. Here 2 is the iteration stage. This is visible to an observer $O_{k, g}$ in $F_{k, g}$. The construction in $F_{k, g}$ is possible because $F_{k, g}$ contains representations of physical theories, including quantum theory, as structures based on $R_{k, g}, C_{k, g}$.

It follows that this construction can be iterated to obtain frames emanating from frames. The iteration or stage number provides a third dimension to the frame field where for each number $j, F_{j, k, g}$ denotes a frame at stage $j$.

There are several different iteration types to consider: a finite number of iterations, a one way infinite number, a two way infinite number, and a finite cyclic iteration. All these types are mathematically possible. They must all be considered as there is no a priori reason to choose one type over another. The different types are illustrated schematically in figures 2 -4.

Figure 2 shows the frame field for a finite number, $n$, of iterations. The iteration paths shown represent two out of an infinite number of paths. Each path segment, shown by an arrow, stands for a quantum theory representation of real and complex numbers described in the frame at the arrow tail. The frame at the arrow head is based on the described quantum theory representation. The iteration direction is shown by the arrows.

Figs. 1 and 2 show the existence of a fixed frame which is an ancestor for all the frames in the field. This is the case even if $n$ is extended to infinity in Fig. 2 to give a one way infinite iteration. Here, too, there is a fixed elementary representation of the real and complex numbers that is external to the whole field.

The two way infinite and cyclic iterations shown in Figs. 3 and 4 are different in this respect. There is no representation of the real and complex numbers that is external for the whole frame field. All are inside some frame as each frame has

\footnotetext{
${ }^{14}$ The axioms for real and complex numbers are respectively those describing a complete, ordered field [36] and an algebraically closed field of characteristic 0 [37.
} 


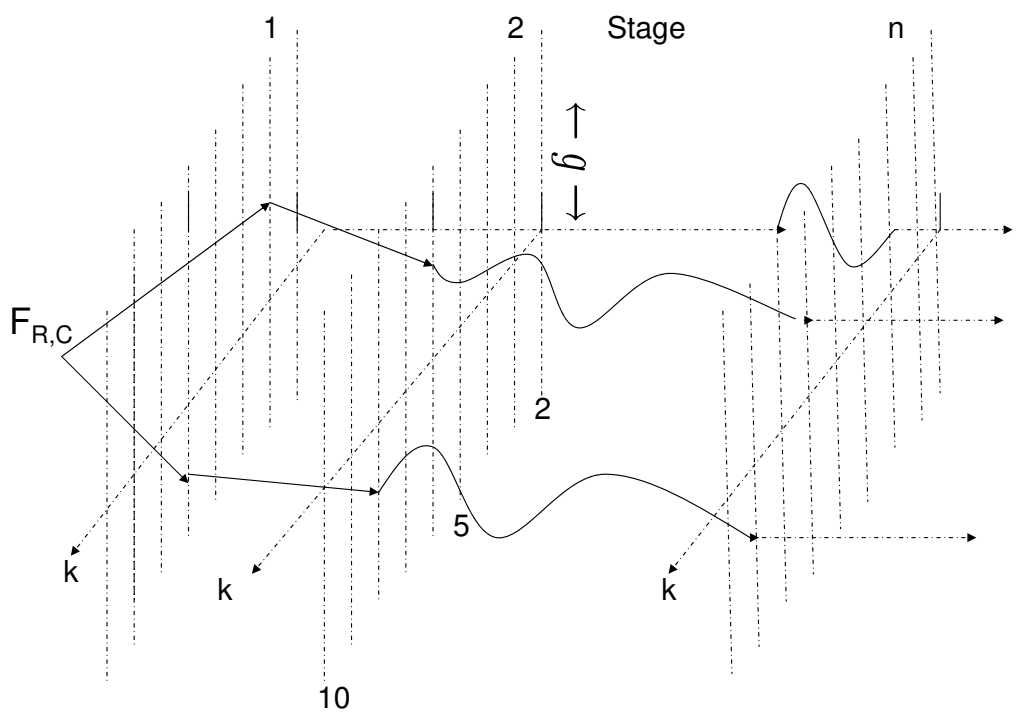

Figure 2: Schematic illustration of a finite number $n$ of frame generations coming from frame $F_{R, C}$. The stage number is given at the top. The direction of the iterations are shown by the solid connected arrows showing sample iteration paths from $F_{R, C}$ through the $k, g$ planes and ending at the $n t h$ plane. The horizontal dashed lines at the right end indicate that in the case of a one-way infinite number of iterations there is no terminal stage $n$ for any finite $n$. See Fig. 1 caption for more details.

parent frames. There is no common ancestor frame.

The path shown in Figure 4 for cyclic iterations is an example of a path with winding number 1 in that it comes to its starting point in one turn around the iteration cylinder. One can, in principle at least have paths with finite winding numbers or even infinite winding numbers in that they never return to the starting point. One hopes to study in the future these types of paths and their dependence on the number of iterations.

The schematic nature of these figures is to be emphasized. Besides showing that two dimensions of the three dimensional frame field are discrete and one, the gauge dimension, is continuous, they are very useful to show what an observer sees in each frame as well as to illustrate the relation between frames in different generations. They are also illustrations of the different iterations that are mathematically possible. Which of the cases is relevant to physics will have to await more work.

The relations between the observers in different frames, described for Fig. 1, is easily extended to multiple iteration stages shown in the other figures. Observers in each frame have in common the property that they can see down the field in the direction of the iterations. That is they can see all their descendent frames, but they cannot see any ancestor frames. They also cannot see any other frame 


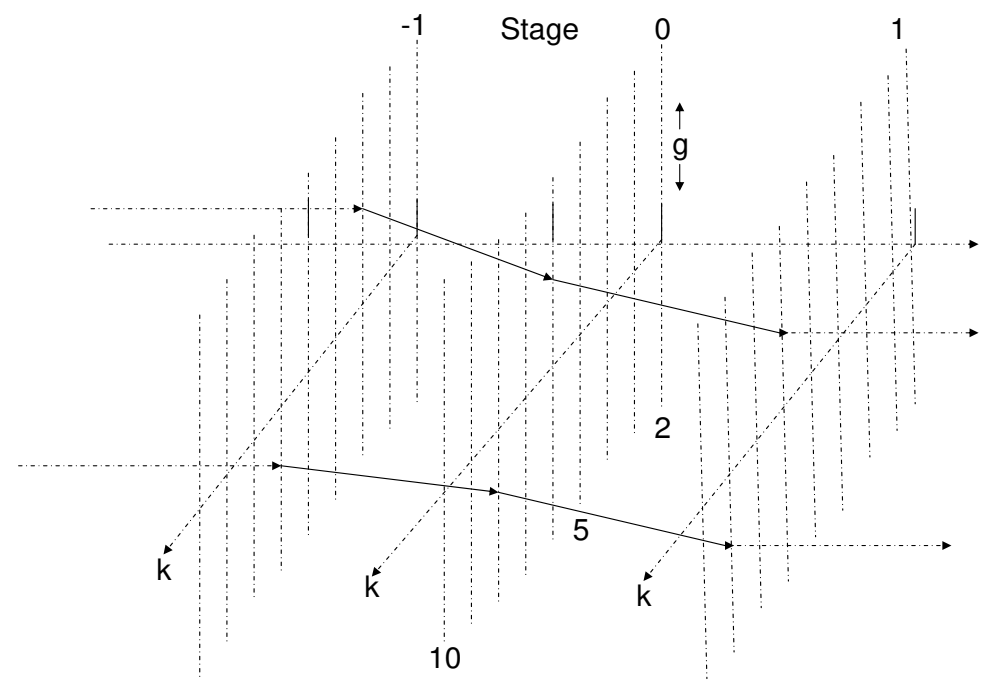

Figure 3: Schematic illustration of a two way infinite number of iterations. Here there is no common ancestral frame as all frames have parent frames and descendent frames. The stage number is given at the top. The direction of the iterations are shown by the solid connected arrows showing a possible path from one stage to the next with no beginning or end.

at the same iteration stage. By "see frames and their relations" is meant that an observer $O_{j, k, g}$ in frame $F_{j, k, g}$ can show the presence of the 2 dimensional frame field $F_{j+1}:\{k, g\} \rightarrow\left\{F_{j+1, k, g}\right\}$. This is what most of this paper has shown. $O_{j, k, g}$ can also shift the derivation by one or more iteration stages to stage $j+2, j+3$ frames, etc. $O_{j, k, g}$ can also see that the $R, C$ representations in these descendent frames have structure as equivalence classes of Cauchy sequences of (pairs of, for $C)$ finite $q_{k}$ string states.

However $O_{j, k, g}$ cannot describe either ancestor frames or other stage $j$ frames. Doing so requires awareness of the real and complex number base of a parent frame. These are not available as they are outside of $F_{j, k, g}$.

It is also clear that no observer in a frame can see the whole frame field. This view is reserved for an observer outside the whole field 15 An observer, $O_{R, C}$, in a common ancestor frame $F_{R, C}$ can see the whole descendent frame field structure. However $O_{R, C}$ cannot tell if there are one or more ancestor stages above.

In many ways this is like the bird (outside the system) and frog (inside the system) views used [8, 9] by Tegmark 16 In effect one has here a hierarchy of bird

\footnotetext{
${ }^{15}$ Here it is assumed that any reader of this paper is outside the whole frame field. Whether this needs to be revised or not must await further work.

${ }^{16}$ These concepts also play a role in mathematical logic in discussions of "absoluteness", i.e. whether or not properties of systems in a model of a set of axioms are preserved when one moves from a view inside the model to one outside the model. 38.
} 


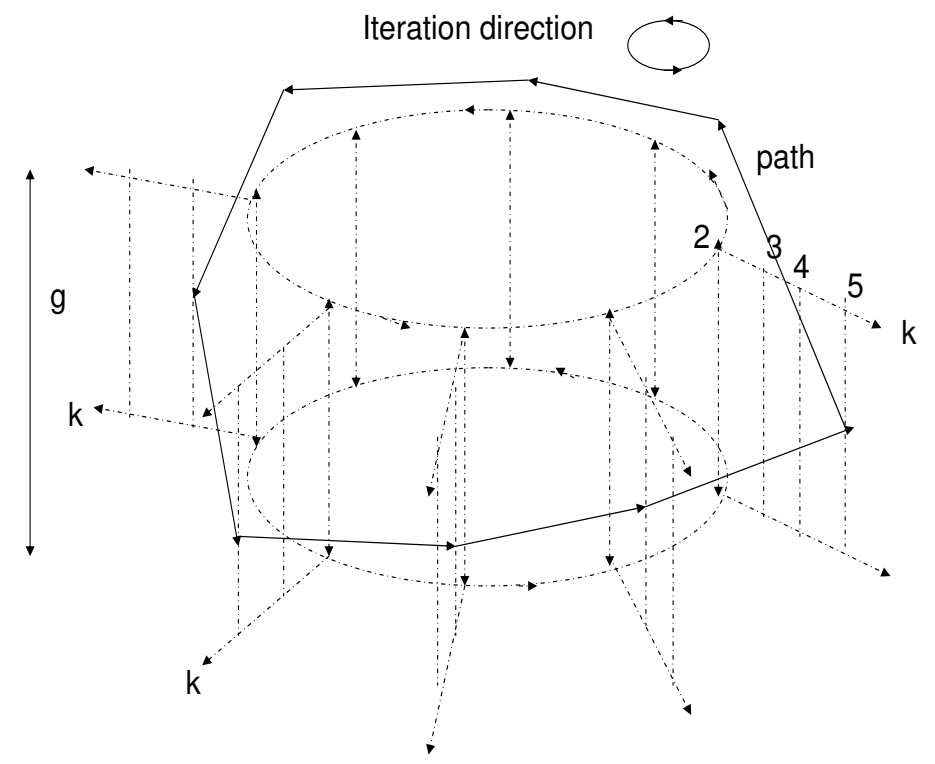

Figure 4: Schematic illustration of a cyclic iteration scheme for a finite number (8) of iterations. There is no common ancestral frame as all frames have parent frames and descendent frames. The stage number is given at the top. The direction of the iterations are shown by the solid connected arrows showing a possible cyclic path. To avoid clutter, the three $g, k$ planes in the back have been suppressed.

and frog views. An observer, $O_{j, k, g}$, in a frame, $F_{j, k, g}$ has a frog view of $F_{j, k, g}$ and of the theories in $F_{j, k, g} . O_{j, k, g}$ sees the real and complex number base, $R_{j, k, g}, C_{j, k, g}$ as elementary. The only relevant properties they have are those derived from the relevant set of axioms. However, $O_{j, k, g}$ has a birds view of all descendent frames in that the relations between all descendent frames are visible.

Cyclic frame iterations present a different situation in that descendant frames are also ancestor frames. Because of this one may have to relax the stipulation that an observer cannot see an ancestor frame. Details of exactly how this would occur are not known at present.

The iteration paths illustrated in the figures give a good representation of what observers in different frames can and cannot see. Each path is a "visibility" path for each frame on the path. If $F_{j, k, g}$ is on a path, then any frame $F_{j^{\prime}, k^{\prime}, g^{\prime}}$ with $j^{\prime}>j$ on the path is a descendent frame and is visible from $F_{j, k, g}$. Frames $F_{j^{\prime}, k^{\prime}, g^{\prime}}$ with $j^{\prime}<j$ are not visible from $F_{j, k, g}$ (except possibly in the cyclic case). The totality of frame visibility from $F_{j, k, g}$ is then given by the descendant frames in all paths passing through $F_{j, k, g}$.

The presence of a three dimensional frame field shows that quantum theory representations have two additional dimensions for the frame field that are not present in classical representations based on kit strings. One is the presence of the freedom of gauge or basis choice. The other is based on the fact that quantum theory, in common with other physical theories, is a theory based on the real 
and complex numbers. The relevant point here is that states of finite strings of qukits are elements of a Fock space which is itself based on the real and complex numbers. This also applies to the states of individual $q_{k}$ which are elements of a $k$ dimensional Hilbert space. Both these spaces are vector spaces over the complex field $C$.

\section{Integration with Physics}

The main problem confronting this work is how to integrate quantum representations of real and complex numbers and fields of iterated reference frames with physics. This relationship would be expected to be an important part of any approach to a coherent theory of physics and mathematics [4, 5], or to any general theory in which physical and mathematical systems are closely related 8 . In particular one may hope that elucidation of this relationship will provide a good foundation to theoretical physics. It also may help to decide which of the different competing theories of quantum gravity, such as loop quantum gravity [40] and string theory [41, is correct.

\subsection{Simple Relations to Physics}

There are some simple ways the work presented here is related to physics. They are called simple only because it is not clear if they would influence the properties of physical theories or affect physics.

One of these, which was noted earlier, is that the choice of number representations as states of single finite $q_{k}$ strings is based on the universality of quantum mechanics as a description of physical systems. Also influencing the choice is the fact that all physical representations of numbers are as states of finite strings of physical systems.

The important role that real and complex numbers have in physics should also be stressed. All theoretical predictions of physical properties of systems are in the form of real numbers as values of physical properties. Also dimensionless constants are presumed to be real numbers. Complex numbers occur as expansion coefficients of superposition states of physical systems and as elements in matrix representations of operators.

Translation of this into the frame field described here has consequences of how the numbers used by physical theories in a frame are seen by observers in different frames. For example an observer $O_{j, k, g}$ in frame $F_{j, k, g}$ sees the real and complex numbers, $R_{j, k, g}, C_{j, k, g}$, as external featureless objects with no properties other than those derived from the real and complex number axioms. Any other properties they may have are not visible to $O_{j, k, g}$.

It follows that, from $O_{j, k, g}^{\prime} s$ viewpoint, all values of physical quantities described or predicted by physical theory representations, as mathematical structures based on $R_{j, k, g}, C_{j, k, g}$ have the same property to $O_{j, k, g}$. This applies to both dimensionless physical quantities such as the fine structure constant and dimensioned quantities such as values of spatial position, distance, momentum, energy, all elements of the spectrum of observables, values of the metric tensor $g_{\mu, \nu}$, etc. 
However an observer $O_{j^{\prime}, k^{\prime}, g^{\prime}}$ in a parent frame $F_{j^{\prime}, k^{\prime}, g^{\prime}}$ where $j^{\prime}=j-1$ sees all elements of $R_{j, k, g}$ as equivalence classes of states of finite $q_{k}$ strings. As a result $O_{j^{\prime}, k^{\prime}, g^{\prime}}$ also sees that all physical quantities described by theories in $F_{j, k, g}$ are equivalence classes of Cauchy sequences of $q_{k}$ string states. To summarize, what $O_{j, k, g}$ sees as elementary featureless objects, are seen by $O_{j^{\prime}, k^{\prime}, g^{\prime}}$ as equivalence classes of Cauchy sequences of states of $q_{k}$ strings.

The same holds for representations of all complex valued physical quantities, such as elements of matrices representing physical transformations, and quantum state expansion coefficients. These quantities in frame $F_{j, k, g}$ are seen by $O_{j^{\prime}, k^{\prime}, g^{\prime}}$ in $F_{j^{\prime}, k^{\prime}, g^{\prime}}$ as equivalence classes of pairs of qukit string states.

In general, all these results on how the values of physical quantities are seen depends on the relation between the frame containing the representations of these quantities and the viewing frame of an observer. They all follow from the observations that in each frame all physical theories are represented as mathematical structures based on the real and complex number base of the frame. How these numbers are seen depends on the relation between the frame based on these numbers and the viewing frame.

Because of much interest in quantum gravity and associated structure of space and time [39], it is worthwhile to consider how a representation of space time and its properties in one frame are viewed from a parent frame. As would be expected, real number values of all physical properties of space and time, which are featureless and elementary in one frame, are viewed as equivalence classes of Cauchy sequences of states of finite $q_{k}$ strings from a parent frame. This applies to distances, angles, coordinate positions, and to values of the metric tensor $g_{\mu, \nu}(x)$. It also applies to matrix representations of space time transformations from one inertial frame to another.

In addition, if one regards the points of the space time manifold as 4 -tuples, $R^{4}$, of the real numbers, then the same arguments hold. In this case an observer in frame $F_{j, k, g}$ sees the points of his own space time manifold, $R_{j, k, g}^{4}$ as 4 -tuples of elementary, featureless points whereas an observer $O_{j^{\prime}, k^{\prime}, g^{\prime}}$ in a parent frame $F_{j^{\prime}, k^{\prime}, g^{\prime}}$ sees the points of $R_{j, k, g}^{4}$ as 4 -tuples of equivalence classes of Cauchy sequences of $q_{k}$ string states. To $O_{j^{\prime}, k^{\prime}, g^{\prime}}$ the space time points in $F_{j, k, g}$ are not featureless as they have structure.

This description of how observers describe space time representations in different frames is valid only if one describes the space time manifold as a 4-tuple of real numbers. For other descriptions, such as discrete space times or space time foams [42, 43, 44, 45, 46, 47, 48, or space represented by spin networks [40, it is not clear if a similar description applies that is based on the relation between the viewing and representation frames.

Another aspect of integrating the frame field with physics is that there is no hint of the frame field structure in the properties of the observed physical universe. This suggests that one should perhaps find some way to collapse the field structure, or at least make the different reference frames appear to be "the same" in some sense. This suggests that one should require that the physical properties of systems represented by frame field elements are frame invariant. That is, they are invariant under transformations from one frame to another. 
One step in this direction is to require that the field structure be such that all frames are equivalent. This would restrict the iteration types to the two way infinite and finite circular ones as they do not have an ancestor frame that is different (from the viewpoint of outside the frame field) from the other reference frames. It also seems appropriate to restrict consideration to the finite cyclic iteration field type, as one way to move toward frame invariance is to reduce the size of the frame field.

The ultimate step in this direction is to reduce the number of iteration stages in a cycle to just one. Whether this is possible or not will have to await future work.

Another approach to reduce the frame field is to eliminate the gauge dimension entirely by requiring the states of the individual $q_{k}$ to be invariant under any basis change. This can be achieved by letting the 0 and 1 states of each $q_{k}$ be represented by different irreducible representations of the gauge group $S U(k)$. One method [31, 49, 50] involves constructing new qukits from the old ones by reducing the product $S U(k) \times S U(k)$ into a sum of irreducible representations and choosing any two representations to represent the 0,1 states of each new $q_{k}$.

Another method [40, 51] uses transformations on the $S U(k)$ group manifold to construct irreducible representations of the group that are invariant under the transformations. In essence this is the method used to construct angular momentum state subspaces labeled by different values of $L$ that are invariant under rotations as transformations on $S O(3)$.

\subsection{Speculative Approaches to Integration with Physics}

So far the approaches to integrating the frame field and quantum representations of real and complex numbers with physics are rather superficial. They do not represent a real integration that treats both physical and mathematical systems together in a coherent way.

How one does this is quite open at present. However one may speculate about various methods to achieve this. One possible way is based on noting that, as units of quantum information, the qukits be considered to be fundamental objects that can represent either components of numbers or physical systems. Whether it represents a number component (digit) or a physical system would depend on how it is viewed.

The details of this would have to be worked out to see if it has merit. However, it is worth noting that this type of dependence already occurs elsewhere in physics, such as the wave-particle and other types of duality. Also the suggestion that one consider the prime number qukits as elementary and the others as composites, may fit in here. In particular the observation is intriguing that, if the prime numbers $p$ are related to particle spin by $p=2 s+1$, there is just one fermion for $s=1 / 2$; all the rest are bosons.

Another approach to constructing a coherent integration with physics is based on the observation that physical theory representations have been inserted into each frame of a completed frame field as mathematical structures based on the real and complex number base of the frame. Instead one may consider involving physical theories in the process of constructing Cauchy sequences, their use to 
represent real and complex numbers, and in properties of the frame field.

In this way physical theories may have input into constructing their real and complex number bases and, conversely, the process of constructing sequences and imposing the Cauchy condition may influence the properties of the physical theories whose base is being constructed. It is even possible that the restrictions imposed by this interlocking process may influence the physical predictions that the theories can make.

It would seem that this approach might be most fruitful in applying it to the cyclic frame fields and possibly those with a very few elements in a cycle. One may speculate that the process of closing the cyclic fields imposes restrictions on the physical theories and numbers involved that influences the values of fundamental constants in the theories or predicted values of physical quantities.

Another approach to integrating this work with physics is based on the possible representation of a sequence $\Psi$ as a $\mathcal{B}_{k, g}$ or, more generally, as an $\mathcal{F}_{k}$ valued quantum field on the nonnegative integers. Then the states of the field at each $n$ are given by $\Psi(n)$. Attention is then restricted to those fields that satisfy the Cauchy condition, i.e. the Cauchy fields.

As was seen, one of the degrees of freedom in representing these fields is the freedom of gauge or basis choice. Changes in gauge are implemented by gauge transformations acting on the fields as shown in Eq. 67, or $\Psi^{\prime}=\mathcal{U}_{k} \Psi$.

This raises the possibility of using the well developed techniques of gauge theories for these fields. For example, one requires that the axioms for the type of numbers being described must be invariant under any gauge transformation. Yet it is clear from their expression in any particular gauge that their expressions transform covariantly under any gauge transformation. The same holds for the expression of the Cauchy condition. This is, ultimately, a consequence of the gauge dependence shown in Eqs. 28 and 27.

One should note that the invariance of the axioms of number theories under gauge transformations also applies to the axioms for any physical theory. The importance of this is stems from the fact that all physical theories have axioms, whether they are implicit or explicitly stated. Without axioms, theories are empty as nothing can be derived or predicted.

For the gauge theory approach one can ask if there is any way to express an action or type of LaGrangian whose invariance under gauge transformations expresses the invariance of the axioms for numbers and for physical theories. If so it may be one way to work towards integrating the results obtained here with physics.

There is an intriguing connection of this approach to the standard model in physics [52, 53. This model is a gauge theory where invariance of the LaGrangian under gauge transformations requires the introduction of fields for the electromagnetic, weak, and strong forces. The invariance is under all gauge transformations in the group $U(1) \times S U(2) \times S U(3)$.

The connection of the standard model to the gauge theory approach to axiom invariance noted above is based on the earlier suggestion that prime number qukits (those whose base $k$ is a prime number) are elementary and the others are composites. Here invariance is under all gauge transformations in $U(1) \times S U(2) \times$ 
$S U(3) \times S U(5) \times \cdots$. The first three groups in the product are the same as those for the standard model. Whether or not the product of groups $S U(p)$ has to include components for all prime numbers or can be cut off is not known at present.

It is not clear if this, or any other speculative approach, will work out. However, these possibilities indicate that there is much work needed to integrate quantum theory representations of numbers and the resulting frame field with physics.

\section{Discussion}

There are some other aspects of this work that should be noted. One is that representation of gauge transformations by one continuous dimension of the frame fields, as in Figs. 1.4, is purely schematic. Nothing is implied about what it means for one gauge $g$ to be close to or far away from another. Indeed it may not be useful or even possible to assign a distance measure to the set of gauges 17

In this connection one should note that the choice of basis sets $\mathcal{B}_{k, g}$ and $\mathcal{B}_{k^{\prime}, g}$ in the spaces $\mathcal{F}_{k}$ and $\mathcal{F}_{k^{\prime}}$ is completely arbitrary 18 There is no way to determine if the $g$ for the $q_{k^{\prime}}$ strings is the same or different than the $g$ for the $q_{k}$ strings.

This is different from the usual situation in physics. There one has an external reference field or frame that can be used to define what it means for a basis of $k$ dimensional systems to be the same or different from a basis of $k^{\prime}$ dimensional systems. Here no such field or common reference frame is present.

In spite of this the two basis sets can be connected by the base changing operator $\tilde{W}_{k^{\prime}, k}$ defined earlier. Recall that if the state $|\gamma, h, s\rangle_{k, g}$ is in the domain of $\tilde{W}_{k^{\prime}, k}$, then the state $\left|\gamma, h, s^{\prime}\right\rangle_{k, g}=\tilde{W}_{k^{\prime}, k}|\gamma, h, s\rangle_{k, g}$ represents the same number in base $k^{\prime}$ as $|\gamma, h, s\rangle_{k, g}$ does in base $k$.

This shows that one can proceed in two ways: Arbitrarily choose both $\mathcal{B}_{k, g}$ and $\mathcal{B}_{k^{\prime}, g}$ and define $\tilde{W}_{k^{\prime}, k}$ to be a map from $\mathcal{B}_{k, g}$ to $\mathcal{B}_{k^{\prime}, g}$. Alternatively choose $\mathcal{B}_{k, g}$ and a definition of $\tilde{W}_{k^{\prime}, k}$ and let $\mathcal{B}_{k^{\prime}, g}$ be the range set of $\tilde{W}_{k^{\prime}, k}$.

These methods work only if $k$ and $k^{\prime}$ have the same prime factors. If this is not the case, one can extend the definition of $\tilde{W}_{k^{\prime}, k}$ by use of definitions to accuracy $\ell$, much as was done for the division operator.

There is another quantum theory representation of real and complex numbers that is based on operators instead of sequences of states of $q_{k}$ strings. To define these operators one replaces the natural number domain of sequences $\Psi$ by states of finite $q_{k}$ strings that represent natural numbers. In this way the sequences $\Psi$ become quantum operators $O$ where

$$
\Psi(n)=O|+, h, n\rangle_{k, g} .
$$

Here $|+, h, n\rangle_{k, g}$ denotes a qukit string state $|+, h, s\rangle_{k, g}$ in $\mathcal{B}_{k, g}$ that represents the number $n$ in base $k 19$ Note that Eq. 93 holds irrespective of whether $\Psi$ is a $\mathcal{B}_{k, g}$

\footnotetext{
${ }^{17}$ Recall that each $g$ is a function from $I \times I$ to a basis set for a $k$ dimensional Hilbert space associated with each element of $I \times I$.

${ }^{18} \mathcal{B}_{k, g}$ and $\mathcal{B}_{k^{\prime}, g}$ are each a set of states of all finite tuples of states of finite length qukit strings for bases $k$ and $k^{\prime}$.

${ }^{19}$ Here for simplicity, $|+, h, s\rangle_{k, g}$ is assumed to be a state with no leading or trailing $0 s$. This can easily be relaxed, if desired.
} 
valued or a more general $\mathcal{F}_{k}$ valued sequence.

One can use Eq. 93 to replace state sequences by operators. The definition of the Cauchy condition can be changed to apply to these operators by quantifying over the states $|+, h, n\rangle_{k, g}$ as natural number representations and replacing the state $\left|\gamma_{j}, h_{j}, s_{j}\right\rangle_{k, g}$ in Eq. 34 by $O|+, h, j\rangle_{k, g}$ and the state $\left.\Psi(j)\right\rangle$ in Eq. 35 by $O|+, h, j\rangle_{k, g}$. Similar replacements are made for $\left|\gamma_{m}, h_{m}, s_{m}\right\rangle_{k, g}$ and $\left.\Psi(m)\right\rangle$. Operators that satisfy the relevant Cauchy condition are denoted here as Cauchy operators.

The rest of the definition of quantum theory representations of real and complex numbers can be taken over to define representations as equivalence classes of Cauchy operators. In that case there does not seem to be a reason why one could not extend the frame field description to apply to Cauchy operators. An observer in a frame would see real valued physical quantities in an immediate descendant frame as equivalence classes of Cauchy operators.

It is clear that there is much to do, both in understanding the representations of theories in the frame fields and in integrating this work with physics. In any case it is seen that quantum representations of real and complex numbers as equivalence

classes of Cauchy sequences of states of qukit strings are different from the usual classical representations. Not only are the quantum equivalence classes larger than the classical ones but the space of representations enjoys two degrees of freedom not present in the space of classical representations. These are the gauge freedom and the iteration stage freedom. The freedom of base choice is present in both quantum and classical representations.

\section{Acknowledgement}

This work was supported by the U.S. Department of Energy, Office of Nuclear Physics, under Contract No. DE-AC02-06CH11357.

\section{References}

[1] E. Wigner, Commum. Pure and Applied Math. 13001 (1960), Reprinted in E. Wigner, Symmetries and Reflections, (Indiana Univ. Press, Bloomington IN 1966), pp222-237.

[2] R. W. Hamming, Amer. Mathematical Monthly, 87,No 2, February, (1980).

[3] The Role of Mathematics in Physical Sciences: Interdisciplinary and Philosophical Aspects, G. Boniolo, P. Budinich, M. Trobok, Eds, Springer Publications, Dordrecht, the Netherlands, 2005.

[4] P. Benioff, Found. Phys. 32,989-1029,(2002) arxiv:quant-ph/0201093.

[5] P. Benioff, Found. Phys. 35, 1825-1856, (2005) arxiv:quant-ph/0403209.

[6] P. Benioff, Intl Jour. Pure, Applied Math. 39, 297-341, (2007), arxiv:quant-ph/0508219. 
[7] P. Benioff, Jour. Phys. Conference Series, 70,012003 (2007), [arXiv: quant $=\mathrm{ph} / 0611139]$; arXiv: quant-ph/0604135.

[8] M. Tegmark, arXiv:0704.0646.

[9] M. Tegmark, Ann. Phys. (NY) 270, 1, (1998) gr-qc/9704009.

[10] D. Finkelstein, Quantum Relativity. Springer-Verlag, Heidelberg (1996).

[11] K.-G. Schlesinger, Journal of Mathematical Physics, 40, 1344-1358 (1999).

[12] S. Titani and H. Kozawa, Internat. Jour. Theoret. Phys. 42, 2575-2602, (2003).

[13] G. Takeuti, Two Applications of Logic to Mathematics Kano Memorial Lecture 3, Princeton University Press, New Jersey, 1978; Quantum set theory, in: E. G. Beltrametti, B. C. van Fraassen, Eds., Current issues in quantum logic, Plenum, pp. 303-322, New York 1981.

[14] M. Ozawa, J. Symbolic Logic 72, 625-648, (2007), [arxiv:math.LO/0604.349].

[15] K. Tokuo, Int. Jour. Theoretical Phys., 43, 2461-2481, 2004.

[16] J. V. Corbett and T. Durt, arXiv:quant-ph/0211180 v1 2002.

[17] M. Davis, Internat. Jour. Theoret. Phys. 16,867-874,(1977).

[18] E. I. Gordon, Soviet Math. Dokl. 18, 1481-1484 (1977).

[19] G. L. Litvinov, V. P. Maslov, and G. B. Shpiz, Archives preprint, quant-ph/9904025, v5, 2002.

[20] A. Doering and C. J. Isham, arXiv:quant-ph/0703060 arXiv:quant-ph/0703062, arXiv:quant-ph/0703064, arXiv:quant-ph/0703066.

[21] J. Krol, "A Model of Spacetime. The Role of Interpretations in Some Grothendieck Topoi", preprint, (2006).

[22] S. Lloyd, Phys. Rev. Lett. 88, 237901, (2002).

[23] P. C. Davies, arXiv:quant-ph/0703041.

[24] S. D. H. Hsu, International J. Modern Phys. A 22, 2895-2907, (2007).

[25] E. Hewitt and K. Stromberg, Real and Abstract Analysis Springer Verlag, Inc. New York, 1965.

[26] J. C. Burkhill and H. Burkhill, A second Course in Mathematical Analysis, Cambridge University Press, Great Britain, 1970.

[27] Y. Aharonov and T. Kaufherr, Phys. Rev. D 30, 368-385, (1984).

[28] S. D. Bartlett, T. Rudolph, and R. W. Spekkens, Phys. Rev. A 70, 032307 (2004). 
[29] P. Benioff, arXiv:0704.3574 [quant-ph].

[30] S. J. van Enk, Phys. Rev. A 71, 032339 (2005).

[31] S.J. van Enk, Phys.Rev. A 73042306 (2006) arxiv:quant-ph/0602079].

[32] D. Poulin and J. Yard, arxiv:quant-ph/0612126.

[33] S. D. Bartlett, T. Rudolph, and R. W. Spekkens, Rev. Mod. Phys. 79, 555-609 (2007).

[34] P. Benioff, Phys. Rev. A 64,052310 (2001) arXiv:quant-ph/0104061v3].

[35] J. Barwise, "An Introduction to First Order Logic" in Handbook of Mathematical Logic, J. Barwise, Ed. Studies in Logic and the Foundations of Mathematics, Vol. 90. North Holland Publishing Co. New york, 1977, pp 5-46.

[36] J. F. Randolph, Basic Real and Abstract Analysis, Academic Press, New York, 1968.

[37] J. R. Shoenfield, Mathematical Logic, Addison Weseley, Reading, Ma. 1967.

[38] T.J. Jech, Lectures in Set theory with Particular Emphasis on the Method of Forcing, Lecture Notes in Mathematics No. 217 Springer Verlag New York 1971.

[39] The Quantum Structure of Space and Time, Proceedings of the 23rd Solvay Conference, Brussels, Belgium, Dec. 1-3, 2005, D. Gross, M. Henneaux, A. Sevrin, Eds., World Scientific Press, New Jersey.

[40] A. Ashtekar and J. Lewandowski, Classical and Quantum Gravity, 21, R53R152, (2004).

[41] B. Zweibach, A First Course in String Theory, Cambridge Univ. Press, New York, N. Y. 2004.

[42] G. 't Hooft, Class. Quant. Grav. 13 1023-1040 (1996) arXiv:gr-qc/9601014.

[43] Y. J. Ng and H. van Dam, Int J. Mod. Phys. A 20,1328-1335, (2005) arXiv:gr-qc/0403057; arXiv:gr-qc/9906003

[44] R. Gambini and J. Pullin, arXiv:gr-qc/0505023

[45] B. G. Sidharth, arXiv:physics0402007.

[46] S. Hossenfelder, arXiv:hep-th/0603032.

[47] A. Kempf and R. Martin, arXiv:0708.0062.

[48] M. Maziashvili, arXiv:0708.1472,

[49] M. S. Byrd, D. Lidar, Lian-Ao Wu, and P. Zanardi, Phys. Rev A 71, 052301 (2005). 
[50] J. Kempe, D. Bacon, D. A. Lidar, and K. B. Whaley, Phys. Rev. A 63, 042307 (2001).

[51] N. Mukunda, G. Marmo, A. Zampini, S. Chaturvedi, and R. Simon, Jour. Math. Phys. 46, 012106 (2005).

[52] S. F. Novaes, arXiv:hep-th/0001283;

[53] A. N. Cottingham and D. A. Greenwood, An Introduction to the Standard Model of Physics, Cambridge University Press, Cambridge, UK, 1998. 